\title{
Measurement of the Semileptonic Branching Fraction of Inclusive $b$ Baryon Decays to $\Lambda$
}

\author{
The OPAL Collaboration
}

\section{Abstract}

We present the first measurement of the ratio $R_{\Lambda \ell}$ defined as

$$
R_{\Lambda \ell}=\mathrm{BR}\left(\Lambda_{\mathrm{b}} \rightarrow \Lambda \ell \mathrm{X}\right) / \mathrm{BR}\left(\Lambda_{\mathrm{b}} \rightarrow \Lambda \mathrm{X}\right)
$$

where $\Lambda_{\mathrm{b}}$ denotes all weakly decaying b baryons and $\ell$ represents the average of electrons and muons. Using all hadronic $Z^{0}$ decay events collected with the OPAL detector near the $\mathrm{Z}^{0}$ resonance, we measure

$$
R_{\Lambda \ell}=(7.0 \pm 1.2 \pm 0.7) \% .
$$

We also measure

$$
\begin{gathered}
f\left(\mathrm{~b} \rightarrow \Lambda_{\mathrm{b}}\right) \cdot \mathrm{BR}\left(\Lambda_{\mathrm{b}} \rightarrow \Lambda \mathrm{X}\right)=(3.93 \pm 0.46 \pm 0.37) \%, \\
f(\mathrm{~b} \rightarrow \mathrm{B}) \cdot \mathrm{BR}(\mathrm{B} \rightarrow \Lambda \mathrm{X})=(1.94 \pm 0.28 \pm 0.24) \%, \text { and } \\
\mathrm{BR}(\mathrm{b} \rightarrow \Lambda \mathrm{X})=(5.87 \pm 0.46 \pm 0.48) \% .
\end{gathered}
$$

In all cases, the uncertainties shown are statistical and systematic, respectively. 


\section{The OPAL Collaboration}

K. Ackerstaff ${ }^{8}$, G. Alexander ${ }^{23}$, J. Allison ${ }^{16}$, N. Altekamp ${ }^{5}$, K. Ametewee ${ }^{25}$, K.J. Anderson ${ }^{9}$, S. Anderson ${ }^{12}$, S. Arcelli ${ }^{2}$, S. Asai ${ }^{24}$, D. Axen ${ }^{29}$, G. Azuelos ${ }^{18, a}$, A.H.Ball ${ }^{17}$, E. Barberio ${ }^{8}$, R.J. Barlow ${ }^{16}$, R. Bartoldus ${ }^{3}$, J.R. Batley ${ }^{5}$, J. Bechtluft ${ }^{14}$, C.Beeston ${ }^{16}$, T. Behnke ${ }^{8}$, A.N.Bell ${ }^{1}$,

K.W.Bell ${ }^{20}$, G. Bella ${ }^{23}$, S. Bentvelsen ${ }^{8}$, P.Berlich ${ }^{10}$, S. Bethke ${ }^{14}$, O. Biebel ${ }^{14}$, A. Biguzzi ${ }^{2}$,

V.Blobel ${ }^{27}$, I.J. Bloodworth ${ }^{1}$, J.E.Bloomer ${ }^{1}$, M.Bobinski ${ }^{10}$, P. Bock ${ }^{11}$, H.M. Bosch ${ }^{11}$,

M. Boutemeur ${ }^{34}$, B.T. Bouwens ${ }^{12}$, S. Braibant ${ }^{12}$, R.M.Brown ${ }^{20}$, H.J.Burckhart ${ }^{8}$, C. Burgard ${ }^{8}$,

R. Bürgin ${ }^{10}$, P.Capiluppi ${ }^{2}$, R.K. Carnegie ${ }^{6}$, A.A.Carter ${ }^{13}$, J.R. Carter ${ }^{5}$, C.Y.Chang ${ }^{17}$, D.G. Charlton ${ }^{1, b}$, D.Chrisman ${ }^{4}$, P.E.L. Clarke ${ }^{15}$, I. Cohen ${ }^{23}$, J.E. Conboy ${ }^{15}$, O.C. Cooke ${ }^{16}$, M. Cuffiani ${ }^{2}$, S. Dado ${ }^{22}$, C.Dallapiccola ${ }^{17}$, G.M.Dallavalle ${ }^{2}$, S. De Jong ${ }^{12}$, L.A. del Pozo ${ }^{8}$, K.Desch ${ }^{3}$, M.S.Dixit ${ }^{7}$, E. do Couto e Silva ${ }^{12}$, M. Doucet ${ }^{18}$, E. Duchovni ${ }^{26}$, G. Duckeck ${ }^{34}$, I.P. Duerdoth ${ }^{16}$, J.E.G.Edwards ${ }^{16}$, P.G.Estabrooks ${ }^{6}$, H.G. Evans ${ }^{9}$, M. Evans ${ }^{13}$, F. Fabbri ${ }^{2}$, P.Fath ${ }^{11}$, F.Fiedler ${ }^{27}$, M.Fierro ${ }^{2}$, H.M.Fischer ${ }^{3}$, R. Folman ${ }^{26}$, D.G.Fong ${ }^{17}$, M. Foucher ${ }^{17}$, A. Fürtjes ${ }^{8}$, P. Gagnon ${ }^{7}$, A. Gaidot ${ }^{21}$, J.W. Gary ${ }^{4}$, J. Gascon ${ }^{18}$, S.M. Gascon-Shotkin ${ }^{17}$, N.I. Geddes ${ }^{20}$, C. Geich-Gimbel ${ }^{3}$, F.X. Gentit ${ }^{21}$, T. Geralis ${ }^{20}$, G. Giacomelli ${ }^{2}$, P. Giacomelli ${ }^{4}$, R. Giacomelli ${ }^{2}$, V. Gibson ${ }^{5}$, W.R. Gibson ${ }^{13}$, D.M. Gingrich ${ }^{30, a}$, D. Glenzinski ${ }^{9}$, J. Goldberg ${ }^{22}$, M.J.Goodrick ${ }^{5}$, W. Gorn ${ }^{4}$, C. Grandi ${ }^{2}$, E. Gross ${ }^{26}$, J. Grunhaus ${ }^{23}$, M. Gruwé ${ }^{8}$, C. Hajdu ${ }^{32}$,

G.G.Hanson ${ }^{12}$, M.Hansroul ${ }^{8}$, M.Hapke ${ }^{13}$, C.K.Hargrove ${ }^{7}$, P.A.Hart ${ }^{9}$, C.Hartmann ${ }^{3}$, M.Hauschild ${ }^{8}$, C.M.Hawkes ${ }^{5}$, R.Hawkings ${ }^{8}$, R.J.Hemingway ${ }^{6}$, M.Herndon ${ }^{17}$, G. Herten ${ }^{10}$, R.D.Heuer ${ }^{8}$, M.D.Hildreth ${ }^{8}$, J.C.Hill ${ }^{5}$, S.J. Hillier ${ }^{1}$, T.Hilse ${ }^{10}$, P.R. Hobson ${ }^{25}$, R.J. Homer ${ }^{1}$,

A.K.Honma ${ }^{28, a}$, D. Horváth ${ }^{32, c}$, R. Howard ${ }^{29}$, R.E.Hughes-Jones ${ }^{16}$, D.E. Hutchcroft ${ }^{5}$,

P. Igo-Kemenes ${ }^{11}$, D.C.Imrie ${ }^{25}$, M.R. Ingram ${ }^{16}$, K.Ishii ${ }^{24}$, A. Jawahery ${ }^{17}$, P.W. Jeffrey ${ }^{20}$, H. Jeremie ${ }^{18}$, M. Jimack ${ }^{1}$, A. Joly ${ }^{18}$, C.R. Jones ${ }^{5}$, G. Jones ${ }^{16}$, M. Jones ${ }^{6}$, R.W.L. Jones ${ }^{8}$, U. Jost ${ }^{11}$,

P. Jovanovic ${ }^{1}$, T.R. Junk ${ }^{8}$, D. Karlen ${ }^{6}$, K. Kawagoe ${ }^{24}$, T. Kawamoto ${ }^{24}$, R.K. Keeler ${ }^{28}$,

R.G.Kellogg ${ }^{17}$, B.W.Kennedy ${ }^{20}$, B.J.King ${ }^{8}$, J. Kirk ${ }^{29}$, S. Kluth ${ }^{8}$, T.Kobayashi ${ }^{24}$, M. Kobel $^{10}$, D.S.Koetke ${ }^{6}$, T.P. Kokott ${ }^{3}$, M.Kolrep ${ }^{10}$, S. Komamiya ${ }^{24}$, T.Kress ${ }^{11}$, P.Krieger ${ }^{6}$, J. von Krogh ${ }^{11}$, P. Kyberd ${ }^{13}$, G.D.Lafferty ${ }^{16}$, H. Lafoux ${ }^{21}$, R.Lahmann ${ }^{17}$, W.P.Lai ${ }^{19}$, D. Lanske ${ }^{14}$, J. Lauber ${ }^{15}$,

S.R. Lautenschlager ${ }^{31}$, J.G. Layter ${ }^{4}$, D. Lazic ${ }^{22}$, A.M.Lee ${ }^{31}$, E. Lefebvre ${ }^{18}$, D. Lellouch ${ }^{26}$, J. Letts ${ }^{2}$, L. Levinson ${ }^{26}$, C. Lewis ${ }^{15}$, S.L.Lloyd ${ }^{13}$, F.K.Loebinger ${ }^{16}$, G.D.Long ${ }^{17}$, M.J. Losty ${ }^{7}$, J. Ludwig ${ }^{10}$, A. Malik ${ }^{21}$, M. Mannelli ${ }^{8}$, S. Marcellini ${ }^{2}$, C.Markus ${ }^{3}$, A.J. Martin ${ }^{13}$, J.P. Martin ${ }^{18}$, G. Martinez ${ }^{17}$, T.Mashimo ${ }^{24}$, W.Matthews ${ }^{25}$, P.Mättig ${ }^{3}$, W.J.McDonald ${ }^{30}$, J. McKenna ${ }^{29}$, E.A.Mckigney ${ }^{15}$, T.J.McMahon ${ }^{1}$, A.I. McNab ${ }^{13}$, R.A. McPherson ${ }^{8}$, F.Meijers ${ }^{8}$, S.Menke ${ }^{3}$, F.S.Merritt ${ }^{9}$, H. Mes ${ }^{7}$, J. Meyer $^{27}$, A.Michelini ${ }^{2}$, G. Mikenberg ${ }^{26}$, D.J.Miller ${ }^{15}$, R. Mir $^{26}$, W.Mohr ${ }^{10}$, A.Montanari ${ }^{2}$, T.Mori ${ }^{24}$, M. Morii ${ }^{24}$, U.Müller ${ }^{3}$, K.Nagai ${ }^{26}$, I. Nakamura ${ }^{24}$, H.A.Neal ${ }^{8}$, B. Nellen ${ }^{3}$, B. Nijjhar ${ }^{16}$,

R. Nisius ${ }^{8}$, S.W. O'Neale ${ }^{1}$, F.G.Oakham ${ }^{7}$, F. Odorici ${ }^{2}$, H.O. Ogren ${ }^{12}$, N.J. Oldershaw ${ }^{16}$, T.Omori ${ }^{24}$, M.J.Oreglia ${ }^{9}$, S. Orito ${ }^{24}$, J.Pálinkás ${ }^{33, d}$, G.Pásztor ${ }^{32}$, J.R.Pater ${ }^{16}$, G.N.Patrick ${ }^{20}$, J. Patt ${ }^{10}$, M.J.Pearce ${ }^{1}$, S. Petzold ${ }^{27}$, P.Pfeifenschneider ${ }^{14}$, J.E.Pilcher ${ }^{9}$, J.Pinfold ${ }^{30}$, D.E.Plane ${ }^{8}$, P. Poffenberger ${ }^{28}$, B. Poli ${ }^{2}$, A.Posthaus ${ }^{3}$, H.Przysiezniak ${ }^{30}$, D.L. Rees ${ }^{1}$, D. Rigby ${ }^{1}$, S. Robertson ${ }^{28}$, S.A.Robins ${ }^{13}$, N. Rodning ${ }^{30}$, J.M.Roney ${ }^{28}$, A. Rooke ${ }^{15}$, E. Ros ${ }^{8}$, A.M.Rossi ${ }^{2}$, M. Rosvick ${ }^{28}$, P. Routenburg ${ }^{30}$, Y. Rozen ${ }^{22}$, K.Runge ${ }^{10}$, O. Runolfsson ${ }^{8}$, U.Ruppel ${ }^{14}$, D.R. Rust ${ }^{12}$, R. Rylko ${ }^{25}$, K.Sachs ${ }^{10}$, E.K.G.Sarkisyan ${ }^{23}$, M.Sasaki ${ }^{24}$, C.Sbarra ${ }^{2}$, A.D.Schaile ${ }^{34}$, O.Schaile ${ }^{34}$, F.Scharf ${ }^{3}$, P.Scharff-Hansen ${ }^{8}$, P.. Schenk ${ }^{27}$, B.Schmitt ${ }^{8}$, S.Schmitt ${ }^{11}$, M.Schröder ${ }^{8}$, H.C.Schultz-Coulon ${ }^{10}$, M.Schulz ${ }^{8}$, M.Schumacher ${ }^{3}$, P.Schütz ${ }^{3}$, W.G.Scott ${ }^{20}$, T.G.Shears ${ }^{16}$, B.C.Shen ${ }^{4}$, C.H.Shepherd-Themistocleous ${ }^{8}$, P.Sherwood ${ }^{15}$, G.P.Siroli ${ }^{2}$, A.Sittler ${ }^{27}$, A.Skillman ${ }^{15}$, A.Skuja ${ }^{17}$, A.M.Smith ${ }^{8}$, T.J.Smith ${ }^{28}$, G.A.Snow ${ }^{17}$, R. Sobie ${ }^{28}$, S.Söldner-Rembold ${ }^{10}$, 
R.W.Springer ${ }^{30}$, M.Sproston ${ }^{20}$, A.Stahl ${ }^{3}$, M.Steiert ${ }^{11}$, K.Stephens ${ }^{16}$, J.Steuerer ${ }^{27}$, B.Stockhausen ${ }^{3}$, D.Strom ${ }^{19}$, F.Strumia ${ }^{8}$, P.Szymanski ${ }^{20}$, R. Tafirout ${ }^{18}$, S.D. Talbot $^{1}$, S. Tanaka ${ }^{24}$, P. Taras ${ }^{18}$, S. Tarem ${ }^{22}$, M. Thiergen ${ }^{10}$, M.A. Thomson ${ }^{8}$, E. von Törne ${ }^{3}$, S. Towers ${ }^{6}$, I. Trigger ${ }^{18}$, T. Tsukamoto ${ }^{24}$, E. Tsur ${ }^{23}$, A.S. Turcot ${ }^{9}$, M.F. Turner-Watson ${ }^{8}$, P. Utzat ${ }^{11}$, R. Van Kooten $^{12}$, G. Vasseur ${ }^{21}$, M. Verzocchi ${ }^{10}$, P. Vikas ${ }^{18}$, M. Vincter ${ }^{28}$, E.H. Vokurka ${ }^{16}$, F. Wäckerle ${ }^{10}$, A. Wagner ${ }^{27}$, C.P.Ward ${ }^{5}$, D.R. Ward ${ }^{5}$, J..J. Ward ${ }^{15}$, P.M.Watkins ${ }^{1}$, A.T. Watson ${ }^{1}$, N.K. Watson ${ }^{7}$, P.S.Wells ${ }^{8}$, N. Wermes ${ }^{3}$, J.S. White ${ }^{28}$, B.. Wilkens ${ }^{10}$, G.W.Wilson ${ }^{27}$, J.A.Wilson ${ }^{1}$, G. Wolf ${ }^{26}$, S. Wotton ${ }^{5}$, T.R. Wyatt ${ }^{16}$, S. Yamashita ${ }^{24}$, G. Yekutieli ${ }^{26}$, V. Zacek ${ }^{18}$

${ }^{1}$ School of Physics and Space Research, University of Birmingham, Birmingham B15 2TT, UK ${ }^{2}$ Dipartimento di Fisica dell' Università di Bologna and INFN, I-40126 Bologna, Italy

${ }^{3}$ Physikalisches Institut, Universität Bonn, D-53115 Bonn, Germany

${ }^{4}$ Department of Physics, University of California, Riverside CA 92521, USA

${ }^{5}$ Cavendish Laboratory, Cambridge CB3 0HE, UK

${ }^{6}$ Ottawa-Carleton Institute for Physics, Department of Physics, Carleton University, Ottawa, Ontario K1S 5B6, Canada

${ }^{7}$ Centre for Research in Particle Physics, Carleton University, Ottawa, Ontario K1S 5B6, Canada ${ }^{8}$ CERN, European Organisation for Particle Physics, CH-1211 Geneva 23, Switzerland

${ }^{9}$ Enrico Fermi Institute and Department of Physics, University of Chicago, Chicago IL 60637, USA

${ }^{10}$ Fakultät für Physik, Albert Ludwigs Universität, D-79104 Freiburg, Germany

${ }^{11}$ Physikalisches Institut, Universität Heidelberg, D-69120 Heidelberg, Germany

${ }^{12}$ Indiana University, Department of Physics, Swain Hall West 117, Bloomington IN 47405, USA

${ }^{13}$ Queen Mary and Westfield College, University of London, London E1 4NS, UK

${ }^{14}$ Technische Hochschule Aachen, III Physikalisches Institut, Sommerfeldstrasse 26-28, D-52056

Aachen, Germany

${ }^{15}$ University College London, London WC1E 6BT, UK

${ }^{16}$ Department of Physics, Schuster Laboratory, The University, Manchester M13 9PL, UK

${ }^{17}$ Department of Physics, University of Maryland, College Park, MD 20742, USA

${ }^{18}$ Laboratoire de Physique Nucléaire, Université de Montréal, Montréal, Quebec H3C 3J7, Canada

${ }^{19}$ University of Oregon, Department of Physics, Eugene OR 97403, USA

${ }^{20}$ Rutherford Appleton Laboratory, Chilton, Didcot, Oxfordshire OX11 0QX, UK

${ }^{21}$ CEA, DAPNIA/SPP, CE-Saclay, F-91191 Gif-sur-Yvette, France

${ }^{22}$ Department of Physics, Technion-Israel Institute of Technology, Haifa 32000, Israel

${ }^{23}$ Department of Physics and Astronomy, Tel Aviv University, Tel Aviv 69978, Israel

${ }^{24}$ International Centre for Elementary Particle Physics and Department of Physics, University of Tokyo, Tokyo 113, and Kobe University, Kobe 657, Japan

${ }^{25}$ Brunel University, Uxbridge, Middlesex UB8 3PH, UK

${ }^{26}$ Particle Physics Department, Weizmann Institute of Science, Rehovot 76100, Israel

${ }^{27}$ Universität Hamburg/DESY, II Institut für Experimental Physik, Notkestrasse 85, D-22607 Ham-

burg, Germany

${ }^{28}$ University of Victoria, Department of Physics, P O Box 3055, Victoria BC V8W 3P6, Canada

${ }^{29}$ University of British Columbia, Department of Physics, Vancouver BC V6T 1Z1, Canada

${ }^{30}$ University of Alberta, Department of Physics, Edmonton AB T6G 2J1, Canada

${ }^{31}$ Duke University, Dept of Physics, Durham, NC 27708-0305, USA

${ }^{32}$ Research Institute for Particle and Nuclear Physics, H-1525 Budapest, P O Box 49, Hungary

${ }^{33}$ Institute of Nuclear Research, H-4001 Debrecen, P O Box 51, Hungary 
${ }^{34}$ Ludwigs-Maximilians-Universität München, Sektion Physik, Am Coulombwall 1, D-85748 Garching, Germany

${ }^{a}$ and at TRIUMF, Vancouver, Canada V6T $2 \mathrm{~A} 3$

${ }^{b}$ and Royal Society University Research Fellow

${ }^{c}$ and Institute of Nuclear Research, Debrecen, Hungary

${ }^{d}$ and Department of Experimental Physics, Lajos Kossuth University, Debrecen, Hungary

\section{Introduction}

Recent measurements have shown that there is a larger than expected lifetime difference between $b$ baryons and $B$ mesons. The average $b$ baryon lifetime is $1.14 \pm 0.08 \mathrm{ps}$ [1] whereas the $\mathrm{B}^{0}$ lifetime is $1.56 \pm \mathbf{0 . 0 6} \mathrm{ps}[1]$. The lifetime difference is expected to arise due to decay amplitudes that directly involve the light quarks (the so-called "non-spectator amplitudes"). So far, however, no theoretical calculation has been able to account for a difference in the lifetime ratio smaller than approximately $0.9[2,3]$, compared to the measured value of $0.731 \pm 0.058$. Since lifetimes and branching fractions are related, an independent way to probe the effects of non-spectator diagrams is to measure the semileptonic branching fractions for the different $b$ hadrons.

Recent calculations which include higher-order perturbative corrections [4, 5] adequately reproduce the experimental results for the $\mathrm{B}$ meson semileptonic branching ratio, $\mathrm{BR}_{S L}^{B}$. These corrections also predict a value for $n_{c}$ consistent with data [5], where $n_{c}$ is the average number of charmed hadrons produced per B decay. So far, no theoretical prediction has been published for the inclusive $\mathrm{b}$ baryon semileptonic branching fraction. Calculations exist for exclusive channels, such as $\operatorname{BR}\left(\Lambda_{\mathrm{b}}^{0} \rightarrow \Lambda_{\mathrm{c}} \ell \nu\right)$ where a prediction has been made for ground state $\mathrm{b}$ baryons within the relativistic quark model [6], placing the semileptonic decay rate for $\Lambda_{\mathrm{b}}^{0} \rightarrow \Lambda_{\mathrm{c}} \mathrm{e} \nu$ at $\left(5.1 \times 10^{10}\right) \mathrm{s}^{-1}$. Using the measured $\Lambda_{\mathrm{b}}^{0}$ lifetime [1], one obtains the exclusive semileptonic branching fraction $(5.8 \pm 0.4) \%$. The error shown reflects only the experimental uncertainty on the $\Lambda_{\mathrm{b}}^{0}$ lifetime.

Given the observed difference in lifetimes between B mesons and b baryons, the semileptonic branching fraction of $b$ baryons is expected to be significantly smaller than for $B$ mesons. The semileptonic branching fraction for $\mathrm{B}$ mesons has been measured at the $\Upsilon(4 S)$ resonance to be $\mathrm{BR}_{S L}^{\mathrm{B}}=(10.43 \pm 0.24) \%$ [1]. Using the results from [1] obtained above the $\mathrm{B}$ meson production threshold, one obtains $\mathrm{BR}_{S L}^{\mathrm{b}}=(11.13 \pm 0.29) \%$. In a more recent measurement, the L3 collaboration reports $\mathrm{BR}_{S L}^{\mathrm{b}}=(10.68 \pm 0.46) \%[7]$ also consistent with the world average. The $b$ superscript indicates that the high-energy data correspond to a mixture of $\mathrm{B}^{ \pm}, \mathrm{B}^{0}, \mathrm{~B}_{\mathrm{s}}$ and $\mathrm{b}$ baryons as opposed to $\mathrm{B}^{ \pm}$and $\mathrm{B}^{0}$ only as at the $\Upsilon(4 S)$ resonance. The production fractions of the different $\mathrm{b}$ hadron species near the $\mathrm{Z}^{0}$ resonance is evaluated to be $(37.8 \pm 2.2) \%$ each for $\mathrm{B}^{ \pm}$and $\mathrm{B}^{0},(11.2 \pm 1.9) \%$ for $\mathrm{B}_{\mathrm{s}}$, and $(13.2 \pm 4.1) \%$ for b baryons[1]. 
An estimate of the semileptonic branching fraction of $b$ baryons can be obtained by scaling the measured semileptonic $B$ meson and generic $b$ hadron branching fractions by the lifetime ratios [1]. Assuming $\Gamma_{\mathrm{sl}}$, the semileptonic width, to be the same for all $\mathrm{b}$ hadrons [5], and given that the semileptonic branching ratio is $\Gamma_{\mathrm{sl}} / \Gamma_{\text {total }}$, one obtains:

$$
\begin{aligned}
& \operatorname{BR}\left(\Lambda_{\mathrm{b}} \rightarrow \ell \mathrm{X}\right)=\frac{\tau_{\Lambda_{\mathrm{b}}}}{\tau_{\mathrm{B}}} \cdot \mathrm{BR}_{S L}^{\mathrm{B}}=(7.5 \pm 0.6) \%, \\
& \operatorname{BR}\left(\Lambda_{\mathrm{b}} \rightarrow \ell \mathrm{X}\right)=\frac{\tau_{\Lambda_{\mathrm{b}}}}{\tau_{\mathrm{b}}} \cdot \mathrm{BR}_{S L}^{\mathrm{b}}=(8.2 \pm 0.6) \%
\end{aligned}
$$

where $\Lambda_{\mathrm{b}}$ denotes the admixture of all weakly decaying $\mathrm{b}$ baryons produced near the $\mathrm{Z}^{0}$ resonance, $\ell$ represents the average of electrons and muons, and $b$ refers to all $b$ hadrons.

This paper describes the measurement of the ratio

$$
R_{\Lambda \ell}=\frac{\operatorname{BR}\left(\Lambda_{\mathrm{b}} \rightarrow \Lambda \ell \mathrm{X}\right)}{\operatorname{BR}\left(\Lambda_{\mathrm{b}} \rightarrow \Lambda \mathrm{X}\right)} .
$$

If non-spectator amplitudes were negligible for all $\mathrm{b}$ hadrons, then $R_{\Lambda \ell}=\mathrm{BR}\left(\Lambda_{\mathrm{b}} \rightarrow \ell \mathrm{X}\right)=$ $\mathrm{BR}(\mathrm{B} \rightarrow \ell \mathrm{X})$. The extent to which $R_{\Lambda \ell}$ and $\mathrm{BR}(\mathrm{B} \rightarrow \ell \mathrm{X})$ differ depends on the magnitude of the non-spectator diagrams in $\mathrm{b}$ hadron decays.

Most of this paper concerns the evaluation of $\mathrm{BR}\left(\Lambda_{\mathrm{b}} \rightarrow \Lambda \mathrm{X}\right)$, the denominator in the calculation of $R_{\Lambda \ell}$. We present a new method which allows the separation of $\Lambda_{\mathrm{b}} \rightarrow \Lambda \mathrm{X}$ events from the main sources of background by imposing more restrictions on the nature of "X", the rest of the decay products found with the $\Lambda$. We require the presence of a $\Lambda$ and another baryon ${ }^{1}$ in the event, and fit the two-dimensional distribution of the momentum spectra of these two baryons to extract the fraction of these events coming from $\Lambda_{\mathrm{b}} \rightarrow \Lambda \mathrm{X}$ and $\mathrm{B} \rightarrow \Lambda \mathrm{X}$ events. These fractions are later used in calculating both $f\left(\mathrm{~b} \rightarrow \Lambda_{\mathrm{b}}\right) \cdot \mathrm{BR}\left(\Lambda_{\mathrm{b}} \rightarrow \Lambda \mathrm{X}\right)$ and $f(\mathrm{~b} \rightarrow \mathrm{B}) \cdot \mathrm{BR}(\mathrm{B} \rightarrow \Lambda \mathrm{X})$. The method used to determine $\mathrm{BR}\left(\Lambda_{\mathrm{b}} \rightarrow \Lambda \ell \mathrm{X}\right)$ in the numerator of $R_{\Lambda \ell}$ has been described elsewhere [8, 9] and will only be mentioned briefly here.

\section{The OPAL detector and Monte Carlo samples}

The OPAL detector is described in reference [10]. The central tracking system is composed of a high-precision silicon microvertex detector, a precision vertex drift chamber, and a large volume jet chamber surrounded by a set of chambers that measure the $z$-coordinate ${ }^{2}(z$ chambers). These detectors are located inside a solenoid. The detectors outside the solenoid consist of a time-of-flight scintillator array and a lead glass electromagnetic calorimeter with a presampler, followed by a hadron calorimeter, consisting of the instrumented return yoke of the magnet, and several layers of muon chambers. Charged particles are identified by their specific energy loss, $\mathrm{d} E / \mathrm{d} x$, in the jet chamber. Further information on the performance of the tracking and $\mathrm{d} E / \mathrm{d} x$ measurements can be found in reference [11].

\footnotetext{
${ }^{1}$ Charge conjugation is implied throughout this paper.

${ }^{2}$ The coordinate system is defined such that the $z$-axis follows the electron beam direction and the $x$-axis pointing towards the centre of the LEP ring. The polar angle $\theta$ is defined relative to the $+z$-axis, and the azimuthal angle $\phi$ is defined relative to the $+x$-axis.
} 
Monte Carlo simulations of 6.5 million inclusive multihadronic $\mathrm{Z}^{0}$ decays and two million heavy flavour hadronic decays $(\mathrm{b} \overline{\mathrm{b}}$ and $c \bar{c})$ are used in this analysis. The multihadronic samples were produced using the JETSET $7.3\left(2.5 \times 10^{6}\right.$ events $)$ and JETSET $7.4\left(4.0 \times 10^{6}\right.$ events) Monte Carlo generators [12] with the fragmentation function of Peterson et al. [13] for heavy quarks. The heavy flavour Monte Carlo events were generated using the JETSET 7.4 version with an updated decay table for $\Lambda_{c}$ decays. All simulated events were passed through the full OPAL detector simulation package [14].

\section{Event selection and method}

This analysis uses data with centre-of-mass energies within $3 \mathrm{GeV}$ of the $\mathrm{Z}^{0}$ peak collected during the 1991-1995 running period when the silicon microvertex detector was operational. Standard hadronic event selection [15] and detector performance requirements select a sample of 3.84 million events. Each event is divided into two hemispheres by the plane perpendicular to the thrust axis. The thrust of the event is required to be greater than 0.8 , and the polar angle of the thrust axis, $\theta_{\mathrm{th}}$, must satisfy $\left|\cos \theta_{\mathrm{th}}\right|<0.75$, ensuring that the event is contained within the central barrel region. Jet finding is done using a cone algorithm [16], with the minimum energy for a jet set to $5.0 \mathrm{GeV}$ and the cone radius set to $550 \mathrm{mrad}$.

Lifetime tagging of b-flavoured events is used to reduce contamination from other quark flavours. Hemispheres were tagged as containing candidate b hadrons ("b-tagged") using secondary vertices reconstructed from charged tracks with the algorithm described in [17]. Properties of such secondary vertices were used as inputs to a neural network algorithm that was trained to select $Z^{0} \rightarrow b \bar{b}$ events. The neural network has seven input parameters, the most important of which are the decay length, its uncertainty and the vertex multiplicity. Based on Monte Carlo studies, we estimate the b-tagging efficiency per event to be $0.442 \pm$ 0.004 with a purity of $(93.7 \pm \mathbf{0 . 5}) \%$ where the errors are from Monte Carlo statistics.

The difficulty in the evaluation of the ratio $R_{\Lambda \ell}=\mathrm{BR}\left(\Lambda_{\mathrm{b}} \rightarrow \Lambda \ell \mathrm{X}\right) / \mathrm{BR}\left(\Lambda_{\mathrm{b}} \rightarrow \Lambda \mathrm{X}\right)$ comes from the evaluation of the denominator, when attempting to distinguish $\Lambda$ 's coming from $\Lambda_{\mathrm{b}}$ decays (which we will refer to as the direct $\Lambda$ 's) from the different sources of background in b-flavoured events. We proceed as follow: If either hemisphere is tagged as containing a primary $b$ flavour quark and one of the hemispheres contains both a $\Lambda$ and an antibaryon $(\overline{\mathrm{p}}$ or $\bar{\Lambda})$, that hemisphere is considered to be a candidate to contain the decay $\Lambda_{\mathrm{b}} \rightarrow \Lambda \mathrm{X}$. The anti-baryon is expected to be produced with the $\Lambda_{\mathrm{b}}$ in order to locally conserve baryon number. This assumption of jet hadronisation with local conservation of baryon number [18] has been verified experimentally [19]. This baryon, which will be referred to as the companion baryon, is required to be identified in order to discriminate against sources of background. The shapes of the momentum distributions for the direct $\Lambda$ and the companion baryon are different for $\Lambda_{\mathrm{b}}$ events and all other b-flavoured events. The B meson decays produce direct $\Lambda$ 's with a momentum spectrum similar to those found in $\Lambda_{\mathrm{b}}$ events, whereas fragmentation $\Lambda$ 's are produced with a softer momentum spectrum. By itself, the $\Lambda$ momentum spectrum is only useful to differentiate between $\mathrm{b}$ hadron decays and fragmentation events. It is possible to separate $\Lambda_{\mathrm{b}} \rightarrow \Lambda \mathrm{X}$ events from $\mathrm{B} \rightarrow \Lambda \mathrm{X}$ events using the momentum spectrum of the companion baryon. For $\Lambda_{\mathrm{b}}$ events, the companion baryon is produced during fragmentation when the $b$ quark hadronises to form the $b$ baryon. 
Hence, the companion baryon has a softer momentum spectrum than for $\mathrm{B} \rightarrow \Lambda \mathrm{X}$ events, where the companion baryon is also a B meson decay product. Both baryons coming from $\mathrm{B}$ meson decays receive a strong boost and have harder momentum spectra than fragmentation particles.

To determine the fraction of candidate events coming from $\Lambda_{\mathrm{b}} \rightarrow \Lambda \mathrm{X}$ decays, we perform a maximum likelihood fit to the two-dimensional $\Lambda$ and companion baryon momentum spectra. This fraction times the number of selected candidates of b-flavoured events containing a direct $\Lambda$ and a companion baryon gives the number of $\Lambda_{\mathrm{b}} \rightarrow \Lambda \mathrm{X}$ events needed at the denominator of $R_{\Lambda \ell}$.

Finally, for the numerator of $R_{\Lambda \ell}$, b-tagged events containing a $\Lambda$ and a prompt lepton (either $e$ or $\mu$ ) are selected without the companion baryon requirement. The ratio of branching ratios is extracted by comparing the number of selected b-tagged events containing a $\Lambda-\ell$ pair to the fraction of events found with a $\Lambda$ and a companion baryon which is attributed to $\Lambda_{\mathrm{b}} \rightarrow \Lambda \mathrm{X}$ decays, as estimated by the fit described above.

\section{1 $\Lambda$ selection}

The $\Lambda$ particle is reconstructed via its $\Lambda \rightarrow \mathrm{p} \pi^{-}$decay [20]. Pairs of oppositely charged tracks are fitted to a common vertex. The track with the larger momentum is assumed to be the proton. The measurement of the ionization energy loss, $\mathrm{d} E / \mathrm{d} x$, is used for particle identification. Both the proton and the pion must have a $\mathrm{d} E / \mathrm{d} x$ measurement compatible with that expected for that particle type. For protons, this requirement discriminates against background from $\mathrm{K}_{\mathrm{s}}^{0} \rightarrow \pi^{+} \pi^{-}$decays. The invariant mass of these candidates is required to be between 1.110 and $1.121 \mathrm{GeV} / c^{2}$. In order to enhance the selection of direct $\Lambda$ 's coming from $\Lambda_{\mathrm{b}} \rightarrow \Lambda \mathrm{X}$ decays and reduce the contribution of $\Lambda$ candidates coming from fragmentation [8], a minimum momentum requirement of $4.5 \mathrm{GeV} / c$ is made. A maximum momentum cut is also applied at $15 \mathrm{GeV} / c$ to restrict the analysis to the well populated region which will later be used for the fit. The fake $\Lambda$ fraction in the data is estimated using both Monte Carlo studies and the $\Lambda$ invariant mass distribution outside the signal region. This fraction is found to be $(5.9 \pm 0.2) \%$ once all selection criteria for the $\Lambda$ and companion baryon are applied, as described next.

\subsection{Companion baryon selection}

The companion baryon can be either a $\bar{\Lambda}$, in which case the same mass selection criteria as above are applied, or an anti-proton. If a $\Lambda \bar{\Lambda}$ pair of candidates is found in the same hemisphere, the higher momentum one is assumed to come from the $\Lambda_{\mathrm{b}}$ decay. All companion $\Lambda$ 's with momentum less than $12 \mathrm{GeV} / c$ are considered. Protons are selected using $\mathrm{d} E / \mathrm{d} x$ measurement information. The proton candidate tracks must have at least one hit in either the silicon microvertex or central vertex chamber, and a sufficient number of hits in the jet chamber for the ionization loss measurement. For these tracks, the measured $\mathrm{d} E / \mathrm{d} x$ is compared with that expected for a given mass hypothesis. The probability that they are consistent with being protons is required to be greater than $1 \%$. The $\mathrm{d} E / \mathrm{d} x$ probability according to 
a Gaussian distribution for the pion hypothesis for the proton candidate must also be less than $1 \%$. Only tracks with momentum in the $0.33-1.4 \mathrm{GeV} / c$ or $3.0-12 \mathrm{GeV} / c$ ranges are retained to exclude the region where the $\mathrm{d} E / \mathrm{d} x$ information cannot be used to discriminate between pions and protons. Proton tracks with momenta greater than $3 \mathrm{GeV} / c$ are furthermore required not to be identified as a lepton using the lepton identification criteria described in the next section.

When two proton candidates are found in the same hemisphere, the track with the higher $\mathrm{d} E / \mathrm{d} x$ proton probability is retained. Most companion baryons are proton candidates $(88.5 \pm$ $0.3) \%$. From the Monte Carlo sample, it is estimated that $(80.6 \pm 0.4) \%$ of these are true protons. The remainder of the companions are $\Lambda$ candidates with an estimated purity of $(90.7 \pm 0.8) \%$. The overall companion baryon sample purity is $(81.8 \pm 0.4) \%$. All errors are statistical only.

\subsection{Lepton identification}

Electron candidates are identified using an artificial neural network based on twelve measured quantities from the electromagnetic calorimeter and the central tracking detector [21]. Muons are identified by associating central detector tracks with track segments in the muon detectors and requiring a position match in two orthogonal coordinates [22]. Electron candidates identified as arising from photon conversions are rejected [21]. Electron and muon candidates are required to have momenta greater than $2 \mathrm{GeV} / c$ and $3 \mathrm{GeV} / c$, respectively.

Prompt lepton candidates are used to identify semileptonic $\mathrm{b}$ baryon decays in the hemisphere containing the $\Lambda$. Cascade leptons $(\mathrm{b} \rightarrow \mathrm{c} \rightarrow \ell)$ are rejected from the sample by

requiring $p_{\mathrm{comb}} \equiv \sqrt{(p / 10)^{2}+p_{t}^{2}}>1.2 \mathrm{GeV} / c$, where $p$ is the lepton momentum and $p_{t}$ is the transverse momentum of the lepton relative to the axis of the jet containing it [23]. This jet axis is calculated including the lepton track. Furthermore, the invariant mass of the $\Lambda$-lepton pair is required to be larger than $2.2 \mathrm{GeV} / c^{2}[8]$ to remove $\Lambda_{\mathrm{c}} \rightarrow \Lambda \ell \mathrm{X}$ events. Using Monte Carlo simulated events, we estimate the average selection efficiency for $\Lambda \ell$ in $\Lambda_{\mathrm{b}} \rightarrow \Lambda \ell \mathrm{X}$ events to be $0.040 \pm 0.002$.

\section{Signal and background estimation}

The methods used to evaluate the respective backgrounds in $\Lambda_{\mathrm{b}} \rightarrow \Lambda \ell \mathrm{X}$ and $\Lambda_{\mathrm{b}} \rightarrow \Lambda \mathrm{X}$ events are described below.

\subsection{Background sources for $\Lambda_{\mathrm{b}} \rightarrow \Lambda \ell \mathrm{X}$}

Right-sign pairs are formed by $\Lambda \ell$ and $\bar{\Lambda} \bar{\ell}$ combinations. $\Lambda$ baryons originating from the fragmentation process rather than directly from heavy hadron decays combined with leptons from semileptonic c- or b-hadron decays are found to be the largest sources of background 
and populate right-sign and wrong-sign combinations roughly equally. Hence, the number of wrong-sign charge candidates is used to estimate the background in the right-sign $\Lambda_{\mathrm{b}} \rightarrow \Lambda \ell \mathrm{X}$ sample. However, in the framework of string fragmentation and the "popcorn" model [18] as implemented in the JETSET Monte Carlo program, it is expected that this background contributes differently to the right-sign and wrong-sign samples. A correction factor is evaluated using the Monte Carlo sample and is applied to the data. This correction factor is similar to the one described in a previous analysis [9] although slightly different since the two analyses were performed with different selection criteria. The number of $\Lambda_{\mathrm{b}} \rightarrow \Lambda \ell \mathrm{X}$ events is obtained by subtracting from the number of right-sign events the number of wrong-sign events divided by the correction factor of $0.91 \pm 0.07$ to account for the imbalance in sign-correlation in the background. The systematic uncertainty quoted includes a contribution accounting for differences obtained with different Monte Carlo generators.

\subsection{Background sources for $\Lambda_{\mathrm{b}} \rightarrow \Lambda \mathrm{X}$}

The background contribution in the $\Lambda_{\mathrm{b}} \rightarrow \Lambda \mathrm{X}$ sample is much more difficult to estimate. We use the Monte Carlo to predict the shape of the signal as well as the backgrounds and fit for these backgrounds in the data. The Monte Carlo events are selected using the same criteria as for data, except that the b-flavour tagging requirement is removed. Instead, $b \bar{b}$ events are selected using information stored at generation. This selection is done to reduce the statistical uncertainties in the Monte Carlo studies and does not influence the shape of the $\Lambda$ and companion baryon momentum spectra, as was verified by comparing the $\Lambda$ momentum spectra for tagged and non-tagged events.

The Monte Carlo events are divided into three categories representing $\Lambda_{\mathrm{b}} \rightarrow \Lambda \mathrm{X}$ events and the two dominant backgrounds.

- The $\Lambda_{\mathrm{b}} \rightarrow \Lambda \mathrm{X}$ sample is formed with Monte Carlo events containing a $\Lambda$ with subsequent decay to $\mathrm{p} \pi$, and a reconstructed companion baryon $(\bar{\Lambda}$ or $\overline{\mathrm{p}})$.

- The second sample contains $\mathrm{B} \rightarrow \Lambda \mathrm{X}$ events having a $\Lambda$ in the final state and a reconstructed companion baryon.

- The third sample contains all other background sources. The biggest contribution $(86 \%)$ comes from $\Lambda$ 's produced in the fragmentation process in $b \bar{b}, c \bar{c}$ and lighter quark flavour events. Other types of backgrounds are included, such as fake $\Lambda$ 's from combinatorial background.

Events are classified in either of the first two categories regardless of the true origin of the reconstructed companion baryon as long as the direct $\Lambda$ is a genuine decay product of a $\mathrm{b}$ hadron. This ensures that all direct $\Lambda$ 's coming from b hadron decays are in one of the first two samples. The relative number of selected light flavours, $c \bar{c}$ and $b \bar{b}$ events in the last sample is adjusted to reflect the flavour composition in the data after the b-tagging requirements are applied.

Using the Monte Carlo simulation, we estimate the fraction of events containing primary $c \bar{c}$ quarks in the final sample after b-tagging to be $(4.3 \pm 0.4) \%$. Contamination from lighter 
flavours after the b-tagging accounts for $(2.0 \pm 0.3) \%$ of the total selected events. These are statistical errors.

\subsection{Background separation method}

As mentioned in section 3 , all b hadron decays produce direct $\Lambda$ 's with a similar momentum spectrum, whereas fragmentation $\Lambda$ 's are produced with a softer momentum spectrum. By itself, the $\Lambda$ momentum spectrum only helps in separating $\mathrm{b}$ hadron decays from fragmentation events. This is illustrated in the left column of figure 1 for Monte Carlo events.

To separate $\Lambda_{\mathrm{b}} \rightarrow \Lambda \mathrm{X}$ events from $\mathrm{B} \rightarrow \Lambda \mathrm{X}$ events, we use the distinctive momentum spectra of the companion baryon. For $\Lambda_{\mathrm{b}}$ events, the companion baryon is produced during fragmentation, and hence has a softer spectrum, whereas for $\mathrm{B} \rightarrow \Lambda \mathrm{X}$ events, both the companion baryon and the direct $\Lambda$ are $\mathrm{B}$ meson decay products. This results in a strong boost, giving them a harder momentum spectra than fragmentation particles. This behaviour is shown in the right column of figure 1 . The joint $\Lambda$ and companion baryon momentum spectra allow the separation of $\Lambda_{\mathrm{b}} \rightarrow \Lambda \mathrm{X}$ events from both types of background events.

\subsection{Extracting the $\Lambda_{\mathrm{b}} \rightarrow \Lambda \mathrm{X}$ fraction}

The fraction of $\Lambda_{\mathrm{b}} \rightarrow \Lambda \mathrm{X}$ events in the denominator of eq. 3 is extracted from the data by performing a binned maximum likelihood fit to the two-dimensional momentum distribution of the $\Lambda$ and the companion baryon. This momentum space is divided into 36 bins ( 6 bins of equal size for each axis). Three parameters are evaluated by the fit: $f_{\Lambda_{\mathrm{b}}}$, the signal fraction $\Lambda_{\mathrm{b}} \rightarrow \Lambda \mathrm{X}, f_{\mathrm{B}}$, the fraction of events coming from $\mathrm{B} \rightarrow \Lambda \mathrm{X}$ decays, and $f_{\text {frag, }}$, the fraction coming from fragmentation and misidentified $\Lambda$ 's. The data are assumed to be a mixture of these three types of events such that the expected number of events in bin $i, N_{i}$, normalised to the total number of selected data events $n_{\text {data }}$, is given by:

$$
N_{i}=\left(f_{\Lambda_{\mathrm{b}}} \frac{N_{\Lambda_{b}}^{i}}{N_{\Lambda_{b}}}+f_{\mathrm{B}} \frac{N_{B}^{i}}{N_{B}}+f_{\text {frag }} \frac{N_{f}^{i}}{N_{f}}\right) \cdot n_{\text {data }}
$$

where $N_{\Lambda_{b}}^{i} / N_{\Lambda_{b}}, N_{B}^{i} / N_{B}$ and $N_{f}^{i} / N_{f}$ represent the fractions of Monte Carlo events in bin $i$ from $\Lambda_{\mathrm{b}}, \mathrm{B}$, and fragmentation plus other background events, respectively. These fractions describe the shape of the two-dimensional momentum distributions for each of the three categories of events. The probability $P_{i}$ that $n_{i}$ data events are observed in bin $i$ when $N_{i}$ are expected is calculated using a Poisson distribution function:

$$
P_{i}=\frac{N_{i}^{n_{i}} e^{-N_{i}}}{n_{i} !}
$$

Finally, the negative log likelihood, calculated from the product of the probabilities $P_{i}$ over all bins, is minimised:

$$
-\ln \mathcal{L}=\sum_{i}\left(N_{i}-n_{i} \ln N_{i}\right)+\text { constant }
$$


OPAL Monte Carlo
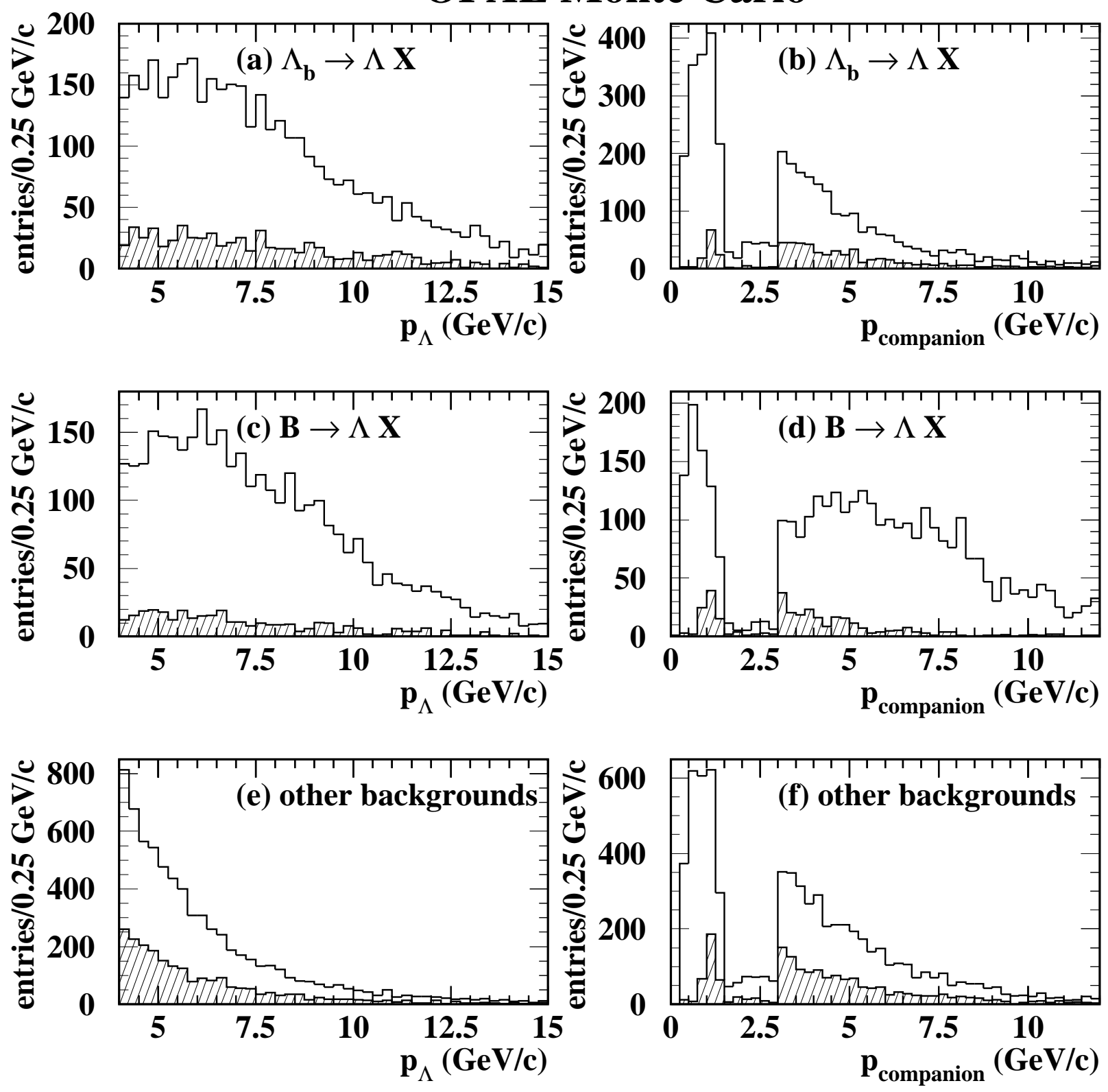

Figure 1: The $\Lambda$ (left column) and the companion baryon (right column) momentum spectra for baryons coming from $\Lambda_{\mathrm{b}}$ decays $(a, b), B$ meson decays $(c, d)$ and from other background events (e,f) are shown for Monte Carlo events. The gap in the momentum spectrum of the companion baryon comes from the proton momentum cut. The hatched areas represent events where the companion baryon is misidentified. The "other backgrounds" sample includes baryons from fragmentation mostly in $\mathrm{b} \overline{\mathrm{b}}$ events, as well as fake $\Lambda$ 's. The spectra shown are obtained after detector simulation and applying all selection criteria. 


\begin{tabular}{|c|c|c|c|}
\hline \multicolumn{4}{|c|}{ Test 1 } \\
\hline & reference & true & fitted \\
\hline$\Lambda_{\mathrm{b}}$ & $27.5 \%$ & $24.6 \%$ & $(27.1 \pm 2.4 \pm 2.5) \%$ \\
B mesons & $30.8 \%$ & $30.7 \%$ & $(30.4 \pm 1.9 \pm 1.9) \%$ \\
other background & $41.7 \%$ & $44.7 \%$ & $(42.4 \pm 2.4 \pm 2.5) \%$ \\
\hline \hline \multicolumn{4}{|c|}{ Test 2} \\
\hline & reference & true & fitted \\
\hline$\Lambda_{\mathrm{b}}$ & $27.5 \%$ & $38.3 \%$ & $(42.4 \pm 3.6 \pm 2.8) \%$ \\
B mesons & $30.8 \%$ & $7.8 \%$ & $(7.6 \pm 2.4 \pm 2.0) \%$ \\
other background & $41.7 \%$ & $53.9 \%$ & $(50.0 \pm 3.6 \pm 2.9) \%$ \\
\hline
\end{tabular}

Table 1: The true composition and the measured fractions from the fitting procedure for two test Monte Carlo samples. The composition of the reference Monte Carlo sample is given in the first column. The uncertainties shown are uncorrelated. The first one reflects the limited sample size of the test sample whereas the second one accounts for the limited number of events in the reference sample. The $\chi^{2} /$ d.o.f. are 25.5 for 33 d.o.f and 36.6 for 32 d.o.f for the first and second tests, respectively. These values of $\chi^{2}$ are only indicators of the goodness-of-fit and are not used in the optimisation process.

\subsection{Testing the $\Lambda_{\mathrm{b}} \rightarrow \Lambda \mathrm{X}$ estimation method with Monte Carlo}

This fitting method does not assume that the composition of the data and Monte Carlo samples is the same. It can also properly distinguish events containing $\Lambda_{\mathrm{b}}$ and B mesons decaying into a $\Lambda$, despite some dilution effect due to the selection of a random track instead of the true companion in about $25 \%$ of $\Lambda_{\mathrm{b}}$ and B meson events. The separation power is tested using Monte Carlo events. The Monte Carlo sample is divided into three independent sets corresponding to the different generator versions described in section 2. For each test, one sample is substituted for 'data' and another sample is used as the reference Monte Carlo. Each sample is compared to the two other Monte Carlo samples and the fitting method is used to evaluate the sample composition, that is, the fit returns the values of $f_{\Lambda_{\mathrm{b}}}$, $f_{\mathrm{B}}$ and $f_{\text {frag }}$ described in equation 4 . The reference Monte Carlo provides the shape of the momentum distributions also described in equation 4 . For each of the six possible tests, the fit estimates the true composition of the test sample to within about 1.5 standard deviations, where the uncertainty is calculated by adding in quadrature the uncorrelated uncertainties due to the limited size of the test sample and the reference sample. Two typical results are shown in table 1 . Note that in the case of test 2 , the fit properly predicts the true test sample composition, despite a very different sample composition in the reference sample.

The fitting procedure also yields consistent results when the event selection criteria for $\Lambda$ 's are modified. The fit is repeated after varying the minimum momentum requirement for $\Lambda$ 's. The fit gives good agreement with the true composition of the Monte Carlo samples in the range of 4.0 to $5.5 \mathrm{GeV} / c$. As the required minimum $\Lambda$ momentum increases, so does the correlation between the $\Lambda_{\mathrm{b}} \rightarrow \Lambda \mathrm{X}$ and the fragmentation background. Beyond $5.5 \mathrm{GeV} / c$, the correlation between $f_{\Lambda_{\mathrm{b}}}$ and $f_{\text {frag }}$ becomes too large and the method loses reliability. The fitting method is not very sensitive to the number of bins used for the fit. The good 


\begin{tabular}{|c|c|}
\hline & \# of event \\
\hline Data & 1595 \\
\hline all Monte Carlo events & 12181 \\
\hline$\Lambda_{\mathrm{b}}$ & 3440 \\
\hline B mesons & 3169 \\
\hline fragmentation and other backgrounds & 5572 \\
\hline fragmentation and other background & 5572 \\
\hline fragmentation in $\mathrm{b} \bar{b}$ & 4115 \\
\hline fragmentation in $c \overline{\mathbf{c}}$ & 501 \\
\hline fragmentation in uds & 234 \\
\hline fake $\Lambda$ 's & 722 \\
\hline
\end{tabular}

Table 2: Numbers of selected events in the data and the Monte Carlo samples. The fragmentation sample contains events with $\Lambda$ 's produced by fragmentation in $b \bar{b}$, $\mathrm{c} \overline{\mathrm{c}}$ and lighter flavour events, as well as fake $\Lambda$ 's.

agreement between fit and true compositions of these test samples under these different changes supports the reliability of the fitting procedure.

The fitting procedure exhibits proper statistical behaviour, as was checked using 1000 variations of the original Monte Carlo momentum distributions. These tests checked the error estimate and that no bias or systematic shift was introduced by the fitting procedure.

\section{Results from the analysis of the data sample}

\section{$5.1 \quad$ The $\Lambda_{\mathrm{b}} \rightarrow \Lambda \mathrm{X}$ sample}

The numbers of $\Lambda_{\mathrm{b}} \rightarrow \Lambda \mathrm{X}$ events selected in the data and in the Monte Carlo samples are shown in table 2. The results of the fit are $f_{\Lambda_{\mathrm{b}}}=0.360 \pm 0.046, f_{\mathrm{B}}=0.192 \pm 0.032$ and $f_{\text {frag }}=0.448 \pm 0.047$, where the statistical uncertainties are shown. The correlation coefficient between $f_{\Lambda_{\mathrm{b}}}$ and $f_{\text {frag }}$ is fairly large $(-0.67)$. There is much less correlation between $f_{\Lambda_{\mathrm{b}}}$ and $f_{\mathrm{B}}(-0.30)$, and between $f_{\mathrm{B}}$ and $f_{\text {frag }}(-0.30)$. The $\chi^{2}$ is 36.8 for 33 degrees of freedom.

In about $25 \%$ of all b hadron events, a random track is selected for companion instead of the baryon produced to locally conserve baryon number. This contamination affects the discrimination power of the fitting procedure by altering the spectrum of true companion baryons for $\mathrm{B}$ mesons. Two thirds of these random tracks have momenta below $2.5 \mathrm{GeV} / c$. We check that selecting a random track instead of the true companion baryon has no effect on the fit result. To do this, we set the minimum momentum requirement for companion baryons to be $2.5 \mathrm{GeV} / c$. The fractions resulting from the fit change by the same amount as the true fractions in Monte Carlo for the same minimum momentum cut. This shows that the analysis selects incorrect companion baryon tracks in a similar proportion in data and Monte Carlo, and that these random tracks have no significant influence on the fit results. 


\subsection{The $\Lambda_{\mathrm{b}} \rightarrow \Lambda \ell \mathrm{X}$ sample}

To estimate the number of $\Lambda_{\mathrm{b}} \rightarrow \Lambda \ell \mathrm{X}$ events in the data, all b-tagged events having a prompt lepton and a $\Lambda$ with momentum greater than $4.5 \mathrm{GeV} / c$ are retained. ${ }^{3}$ No companion baryon is required for this sample since requiring a prompt lepton is sufficient to select $\Lambda_{\mathrm{b}} \rightarrow \Lambda \ell \mathrm{X}$ events $[8,9]$. In the data, 298 events are selected, 215 of which have the rightsign lepton charge and baryon number correlation, and 83 have the wrong-sign. Of these events, 133 contained an electron, and 165 a muon. After correcting for the right-sign wrongsign imbalance as described in section 4.1, the number of $\Lambda_{\mathrm{b}} \rightarrow \Lambda \ell \mathrm{X}$ events in the data is found to be $144.9 \pm 17.3 \pm 11.1(64.8 \pm 11.5 \pm 5.0$ electrons and $80.3 \pm 12.8 \pm 6.1$ muons $)$. The second uncertainty comes from the estimate of the correction factor.

\section{Corrections and sources of systematic uncertainties}

The most important contributions to the systematic uncertainty for $R_{\Lambda \ell}=\mathrm{BR}\left(\Lambda_{\mathrm{b}} \rightarrow \Lambda \ell \mathrm{X}\right) /$ $\operatorname{BR}\left(\Lambda_{\mathrm{b}} \rightarrow \Lambda \mathrm{X}\right)$ are associated with the estimate of the $\Lambda_{\mathrm{b}}$ fraction in the $\Lambda \mathrm{X}$ sample. The uncertainty arises from the proper Monte Carlo simulation of the $\Lambda$ and the companion baryon spectra, and the limited number of Monte Carlo events used for the fitting procedure. In the next three sections, we compare data and Monte Carlo for each spectrum used in the extraction of the fraction of $\Lambda_{\mathrm{b}}$ events.

Throughout this section, unless otherwise specified, the systematic uncertainty is taken to be the size of the excursion from the central value when the studied parameter is allowed to vary within some limits. In the case where a significant difference between Monte Carlo and data is observed, a correction is applied. The final values for the systematic uncertainties and shifts due to corrections are listed in table 3 . An additional source of systematic uncertainty that does not contribute to the calculation of $R_{\Lambda \ell}$ but is needed to evaluate $f\left(\mathrm{~b} \rightarrow \Lambda_{\mathrm{b}}\right) \cdot \operatorname{BR}\left(\Lambda_{\mathrm{b}} \rightarrow\right.$ $\Lambda \mathrm{X})$ and $f(\mathrm{~b} \rightarrow \mathrm{B}) \cdot \mathrm{BR}(\mathrm{B} \rightarrow \Lambda \mathrm{X})$ is given in the last subsection.

\section{1 $\Lambda$ spectrum from $\Lambda_{b}$ decays}

To check that the Monte Carlo properly simulates the $\Lambda$ momentum spectrum of $\Lambda_{\mathrm{b}} \rightarrow \Lambda \mathrm{X}$ baryon decays as shown in figure 1(a), we compare the spectrum of $\Lambda$ 's produced in $\Lambda_{\mathrm{b}}$ semileptonic decay between data and Monte Carlo simulation. The Monte Carlo $\Lambda$ momentum spectrum from $\Lambda_{\mathrm{b}} \rightarrow \Lambda \mathrm{X}$ decays is compared to that of $\Lambda$ 's coming from $\Lambda_{\mathrm{b}} \rightarrow \Lambda \ell \mathrm{X}$ decays to ensure that the test is meaningful. Figure 2(a) shows that the Monte Carlo predicts no measurable difference between the $\Lambda$ spectrum in $\Lambda_{\mathrm{b}} \rightarrow \Lambda \ell \mathrm{X}$ and $\Lambda_{\mathrm{b}} \rightarrow \Lambda \mathrm{X}$ events.

The $\Lambda_{\mathrm{b}} \rightarrow \Lambda \ell \mathrm{X}$ events are selected as described in section 3.3, except that b-tagging is not imposed and the minimum $\Lambda$ momentum is reduced to $4 \mathrm{GeV} / c$ in order to increase statistics. The spectrum from wrong-sign lepton-baryon combinations is subtracted from

\footnotetext{
${ }^{3}$ The b-tagging requirement is imposed on these events to maintain uniformity when selecting $\Lambda_{\mathrm{b}} \rightarrow \Lambda \mathrm{X}$ and $\Lambda_{\mathrm{b}} \rightarrow \Lambda \ell \mathrm{X}$ events. Monte Carlo simulation studies showed that the b-tagging requirement does not distort the $\Lambda$ momentum spectrum.
} 
the right-sign spectrum to form the "direct" spectrum shown in figure 2(b) for data and Monte Carlo. The measured mean $\Lambda$ momentum in data is $(7.32 \pm 0.13) \mathrm{GeV} / c$ compared to $(7.59 \pm 0.09) \mathrm{GeV} / c$ in Monte Carlo after the wrong-sign subtraction. The $\chi^{2}$ in $\Lambda \ell$ events between Monte Carlo and data is measured to be 14.5 for 17 d.o.f. in figure 2(b). The observed difference in the mean $\Lambda$ momentum in $\Lambda \ell$ events between Monte Carlo and data is corrected by scaling the $\Lambda$ momenta for Monte Carlo events in the $\Lambda_{\mathrm{b}} \rightarrow \Lambda \mathrm{X}$ sample by 0.95 . This slightly improves the overall agreement between Monte Carlo and data. When the fit is redone with this modified Monte Carlo $\Lambda$ momentum spectrum, the value of $f_{\Lambda_{\mathrm{b}}}$ changes from 0.360 to 0.401 . This is taken into account by including a correction to the fitted fraction of $+\mathbf{0 . 0 4 1}$. An uncertainty in the $\Lambda$ momentum scale factor of \pm 0.02 is assumed, corresponding to one standard deviation in the measured average momentum for $\Lambda$ 's coming from $\Lambda_{\mathrm{b}}$ decays in data. When the fit is redone with a scale factor varying between 0.93 and 0.97 , the fitting procedure evaluates $f_{\Lambda_{\mathrm{b}}}$ to be between 0.385 and 0.426 , which results in an uncertainty in $f_{\Lambda_{\mathrm{b}}}$ of $\pm \mathbf{0 . 0 2 1}$. This correction also takes into account possible differences in the $\Lambda$ momentum spectra between data and Monte Carlo coming from incorrect modelling of the relative population of excited $b$ baryon states.

In addition to the test shown in figure 2(a), we also use Monte Carlo events to determine the effect of the $\Lambda_{\mathrm{b}}$ decay multiplicity on the $\Lambda$ momentum spectrum since that could lead to differences in the momentum spectrum of $\Lambda$ 's from semileptonic $\Lambda_{\mathrm{b}}$ decays compared to all $\Lambda_{\mathrm{b}}$ events. We examine all $\Lambda_{\mathrm{b}}$ decays and compare the average $\Lambda$ momentum in hadronic and semileptonic decays, looking for a difference in average momenta. The average momentum for a $\Lambda$ from $\Lambda_{\mathrm{b}} \rightarrow \Lambda \pi \pi \pi$ is measured to be about 0.97 times that from a semileptonic decay, well within the range of variation investigated. Hence, no further correction or systematic uncertainty is applied.

The momentum spectrum of the $\Lambda_{\mathrm{b}}$ decay products depends both on the model used to simulate the decay and on the $\Lambda_{\mathrm{b}}$ polarisation. We use two additional Monte Carlo samples to check the dependence of the $\Lambda$ spectrum on the decay model and $\Lambda_{\mathrm{b}}$ polarisation. A first set of $\Lambda_{\mathrm{b}} \rightarrow \Lambda \ell \mathrm{X}$ events $^{4}$ is selected from a separate Monte Carlo sample [9] where the b baryon decay is simulated using a different decay model [24]. In this model, the momentum distribution of the $b$ baryon decay products depends strongly on the form factors used to describe the energy transfer from the $b$ baryon to the $c$ baryon. The particular choice of form factors [25] used in generating these events results in a softer momentum spectrum for the $\mathrm{c}$ baryon and therefore a softer $\Lambda$ spectrum. A second set of Monte Carlo events was generated using the same decay model and adding maximum polarisation to the $\Lambda_{\mathrm{b}}$ baryons.

In each case, the $\Lambda$ momentum spectrum for these Monte Carlo events is compared with the spectrum found for semileptonic $\Lambda_{\mathrm{b}}$ events in the data, shown in figure 2 . The differences observed are within the limits allowed for the uncertainty in the shape of the $\Lambda$ spectrum in $\Lambda_{\mathrm{b}}$ decays. Hence, no additional correction or systematic uncertainty is needed to account for the effects of decay model or polarisation.

\footnotetext{
${ }^{4}$ Form factors and polarisation have been simulated only in semileptonic decays.
} 

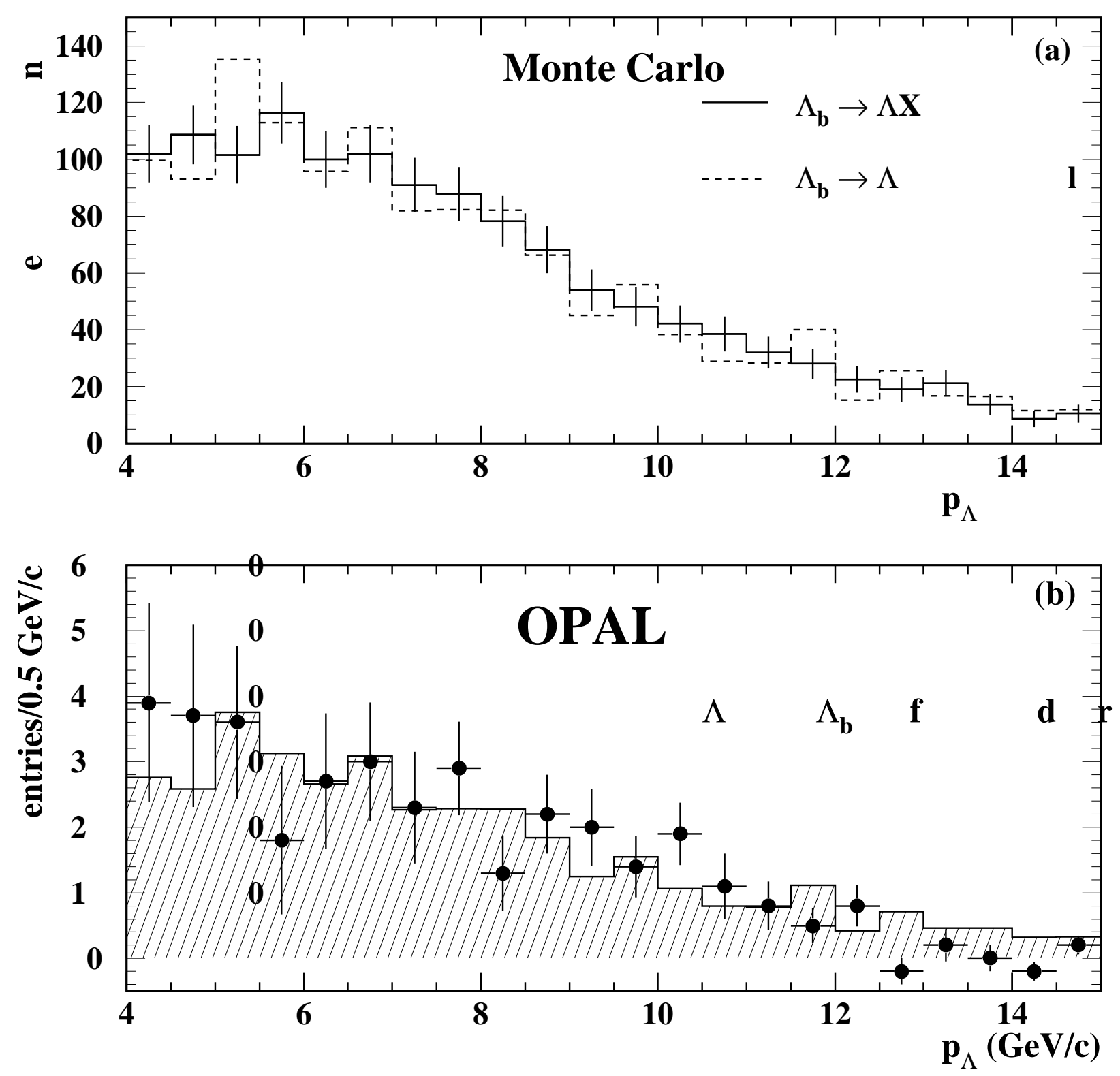

Figure 2: (a) Two types of events from the Monte Carlo simulation are compared: The direct $\Lambda$ 's produced in the $\Lambda_{\mathrm{b}} \rightarrow \Lambda \ell \mathrm{X}$ decays (dashed line) compared to $\Lambda$ 's from $\Lambda_{\mathrm{b}} \rightarrow \Lambda \mathrm{X}$ decays (solid line). (b) The momentum spectrum of direct $\Lambda$ 's produced in semileptonic $\Lambda_{\mathrm{b}} \rightarrow \Lambda \ell \mathrm{X}$ events for data (points) and Monte Carlo (already shown in (a)) (hatched histogram) after wrong-sign subtraction. 


\subsection{Fragmentation spectrum}

This section describes studies that check the Monte Carlo spectra for baryons created in the fragmentation process, such as those shown in figures $1(\mathrm{~b}),(\mathrm{e})$ and (f). We use two different methods: the first one is a direct but low statistics technique, whereas the second is an indirect approach with the advantage of high statistics.

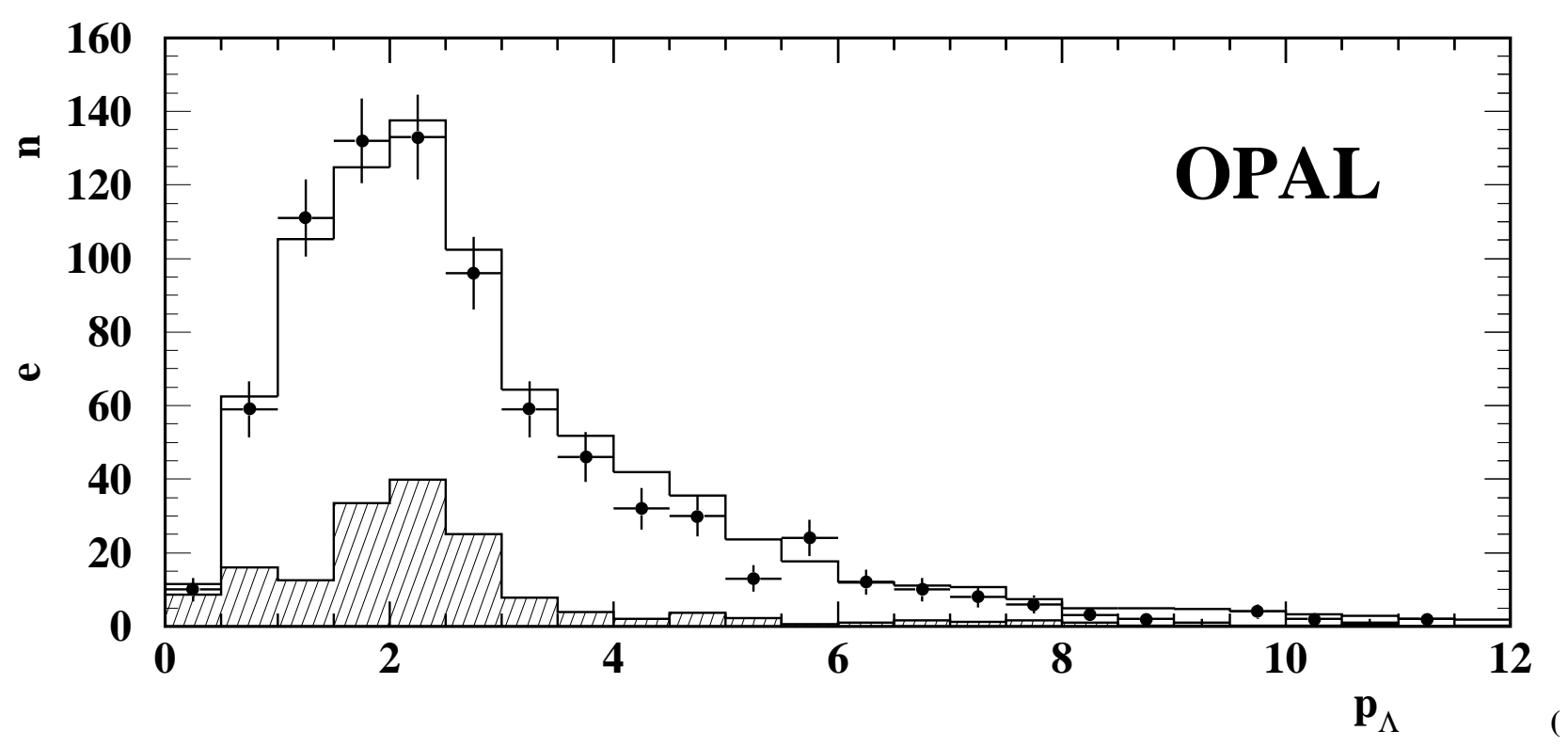

Figure 3: The momentum spectrum of $\Lambda$ 's selected with a prompt anti-lepton in $b$ tagged events. The points represent the data and the histograms show the spectra for Monte Carlo events after they were normalised to the number of data events. The hatched histogram corresponds to contamination from misreconstructed $\Lambda$ 's.

To test the spectrum shown in figure 1(b) (baryons produced from fragmentation in a $\Lambda_{\mathrm{b}}$ event), we look for a companion baryon in semileptonic $\Lambda_{\mathrm{b}}$ events and compare the data directly to the Monte Carlo after subtracting the wrong-sign $\Lambda$-lepton combinations from the right-sign combinations. According to Monte Carlo studies, (95 \pm 1$) \%$ of $\Lambda$ 's found in a wrong-sign $\Lambda$-lepton combination are from fragmentation. Of those events coming from fragmentation, $(82 \pm 3) \%$ contain a properly identified companion baryon. Only 130 events are found in the data sample containing both a $\Lambda$-lepton pair with the wrong sign combination and a companion baryon even after removing the b-tagging requirement. Given these statistical limitations, we can only infer that there is no gross problem in the Monte Carlo simulation of the fragmentation spectrum displayed in figures 1 (b), (e) and (f).

For a second but more indirect check of the fragmentation baryon momentum simulation, the shape of the momentum spectrum for baryons coming from fragmentation is extracted from the data and compared with Monte Carlo events. These baryons are either created during the fragmentation process in $b \bar{b}$ events or are produced to conserve baryon number in $\Lambda_{\mathrm{b}}$ production. Such events are selected by requiring that either hemisphere satisfies the b-tagging requirements described earlier. Each hemisphere is then searched for a prompt lepton (as defined in section 3.3) and an anti-baryon (either $\bar{\Lambda}$ or $\bar{p}$ ). In the Monte Carlo 
simulation, $(83.8 \pm 0.2) \%$ of these prompt leptons originate from a b quark decay. Only events with the wrong-sign combination (i.e. a lepton and an anti-baryon pair) are retained in order to preferentially select baryons produced in the fragmentation and to reject baryons coming directly from $\mathrm{b}$ hadron decay. Using the Monte Carlo, we estimate that $(91.8 \pm 0.5) \%$ of the true $\Lambda$ 's so selected are baryons coming from the fragmentation process. The spectrum of these $\Lambda$ 's is shown in figure 3 , where the hatched region represents the misidentified particles.

For the proton sample, the simpler selection ${ }^{5}$ requirements imposed for this test lead to higher background contamination by misidentified kaons. By rejecting kaons using a more stringent $\mathrm{d} E / \mathrm{d} x$ requirement than required for the main analysis, we can select a purer sample of protons. The momentum spectrum of these proton candidates is shown in figure 4 for two separate momentum ranges. Only anti-protons are shown in figure 4(a) to reduce contributions from secondary interactions which produce mainly protons below $1 \mathrm{GeV} / c$. We estimate that $(98.1 \pm 0.2) \%$ of the protons shown in figure $4(\mathrm{a})$ and $(92.4 \pm 1.2) \%$ of those shown in (b) are baryons created during the fragmentation process. A systematic uncertainty from this source is ascribed by modifying the fragmentation spectrum for Monte Carlo events and refitting the data. For $\Lambda$ 's, the mean momentum in the data is $\bar{p}_{\text {data }}=(2.80 \pm 0.06) \mathrm{GeV} / c$ compared to $\bar{p}_{\mathrm{MC}}=(2.98 \pm \mathbf{0 . 0 3}) \mathrm{GeV} / \mathrm{c}$ in the Monte Carlo. In figure 4(a), the mean momentum for anti-protons from the data is $\bar{p}_{\text {data }}=(0.913 \pm 0.008) \mathrm{GeV} / c$ whereas for Monte Carlo events, $\bar{p}_{\mathrm{MC}}=(0.935 \pm 0.004) \mathrm{GeV} / c$, when including all background contaminants. Above 3 $\mathrm{GeV} / c$ in figure $4(\mathrm{~b})$, we obtain $\bar{p}_{\text {data }}=(5.80 \pm 0.13) \mathrm{GeV} / c$ and $\bar{p}_{\mathrm{MC}}=(5.91 \pm 0.08) \mathrm{GeV} / c$. The small observed difference in the average proton momentum is corrected for in the Monte Carlo by applying a multiplicative factor of 0.976 to the momentum of all baryons coming from fragmentation. When refitting with these modified momenta, we observe a shift of the central value for $f_{\Lambda_{\mathrm{b}}}$ of $-\mathbf{0 . 0 0 5}$. An uncertainty of \pm 0.010 is taken on the correction factor, which leads to a systematic uncertainty of $\pm \mathbf{0 . 0 0 3}$ on $f_{\Lambda_{\mathrm{b}}}$. The shifts and uncertainties are shown in table 3 for the three fit parameters.

\section{3 $\Lambda$ 's coming from $B$ meson decays}

We compare the momentum spectrum of $\Lambda$ 's coming from B meson decays in the simulated sample (such as those shown in figure $1(\mathrm{c})$ and (d)) to the spectrum for $\mathrm{B} \rightarrow \Lambda \mathrm{X}$ events measured by the CLEO collaboration [26]. At the $\Upsilon(4 S)$ resonance, B mesons are essentially produced at rest. We use the shape of the distribution for $x_{p}$, the fraction of the maximum momentum carried by the $\Lambda$, as defined by CLEO. The maximum momentum is determined by the difference between the beam energy at the $\Upsilon(4 S)$ resonance and the mass of the $\Lambda$. We reweight the Monte Carlo events to simulate the $x_{p}$ distribution observed by CLEO. There is very little difference in the average momentum of $\Lambda$ 's $(1.6 \%)$ between weighted and unweighted events for the $\Lambda$ momentum distribution. We estimate the systematic uncertainty by using the weighted events to perform the fit. There is no effect on the fitted value for $f_{\Lambda_{\mathrm{b}}}$ and a small change for $f_{\mathrm{B}}$ and $f_{\text {frag }}$, hence the small contributions to the systematic uncertainties shown in table 3 .

\footnotetext{
${ }^{5}$ No direct $\Lambda$ is required for this sample, as was the case when selecting companion protons for the main analysis.
} 

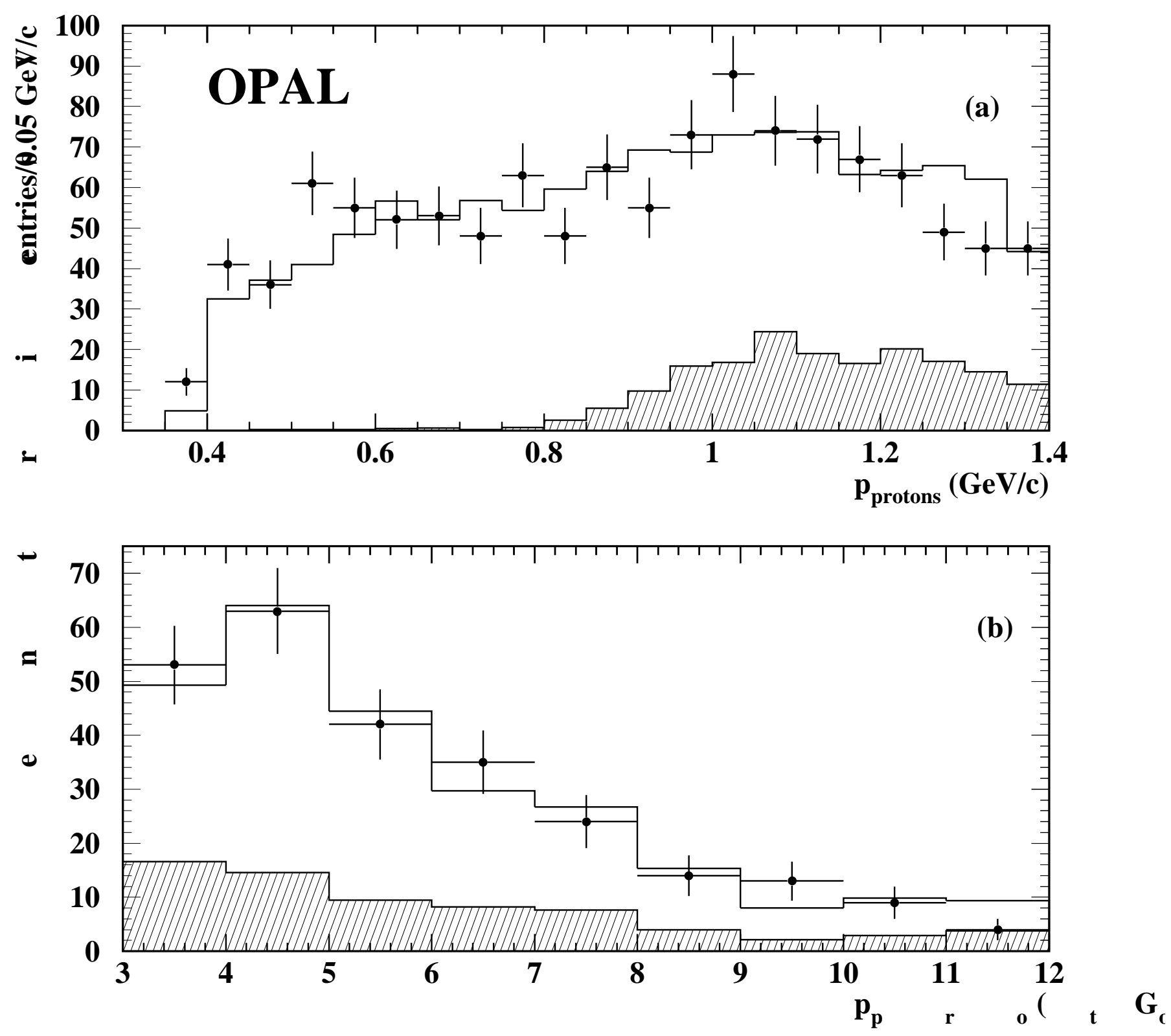

Figure 4: Comparison of the momentum spectrum in data (points) and Monte Carlo (histograms) for a sample of protons selected with a prompt anti-lepton in b-tagged events and after rejecting tracks consistent with being a kaon. The spectra are shown for two different ranges: (a) 0.33-1.4 GeV/c (anti-protons only) and (b) 3.0-12 $\mathrm{GeV} / \mathrm{c}$. The hatched histogram shows the level of contamination from misidentified particles. 


\subsection{Protons coming from B meson decays}

For companion protons produced in the $\mathrm{B} \rightarrow \Lambda \mathrm{X}$ decays, we do not have a direct way of comparing the Monte Carlo simulation with the data. Based on the good agreement with data found for companion $\Lambda$ 's in these decays and the general agreement of our Monte Carlo simulation for the momentum spectrum of all protons found in b-tagged events containing a prompt lepton, we estimate the contribution to the systematic uncertainty by modifying the momentum of companion protons by a multiplicative factor ranging from 0.95 to 1.05 and refitting. The corresponding variations in the estimate of the sample composition are shown in table 3 .

\subsection{The b hadron momentum spectrum}

The $\Lambda$ and companion baryon momenta are also modified to evaluate the effects of a slightly different $b$ hadron momentum spectrum on the fit results. The average $b$ hadron momentum has been measured by OPAL [9] to be $(33.9 \pm 0.2) \mathrm{GeV} / c$, compared to $33.7 \mathrm{GeV} / c$ in the Monte Carlo simulation. Accordingly, variations in the momentum of the $\Lambda$ 's coming from b hadron decays ( $\mathrm{B}$ mesons and $\Lambda_{\mathrm{b}}$ 's) by $\pm 0.6 \%$ were investigated. The fit is repeated with these modified momenta, giving a contribution of \pm 0.003 to the systematic uncertainty from this source. This uncertainty can be expected to account for possible differences in the baryon spectra between data and Monte Carlo simulation due to the parametrisation of the fragmentation process.

\subsection{Background in the $\Lambda$ sample}

The background in the $\Lambda$ sample is compared between data and Monte Carlo using the sidebands of the (p- $\pi)$ invariant mass distribution. A fake $\Lambda$ fraction of $(5.9 \pm 0.2) \%$ is found in the $\Lambda_{\mathrm{b}} \rightarrow \Lambda \mathrm{X}$ region for Monte Carlo, in agreement with the rate of $(5.9 \pm 1.1) \%$ observed in the data. We evaluate the contribution to the systematic uncertainty by adding background events to the Monte Carlo sample so as to raise the fake $\Lambda$ rate to $7 \%$, corresponding to one standard deviation in the fake rate measured in data. When refitting, the value of $f_{\Lambda_{\mathrm{b}}}$ changes from 0.360 to 0.358 . The difference is taken to be the size of the systematic uncertainty, that is $\pm \mathbf{0 . 0 0 2}$.

\subsection{Finite Monte Carlo sample size}

The finite Monte Carlo sample size introduces an additional uncertainty in the fit result. This systematic uncertainty is evaluated separately using a set of 1000 "toy" Monte Carlo samples. The number of Monte Carlo events found in each momentum bin is allowed to fluctuate around the initial central value according to Poisson statistics. Each sample is used to fit the data sample. The spread in the distribution of results for $f_{\Lambda_{b}}$ for these 1000 separate trials is a measure of the systematic uncertainty introduced in the fit due to the 
limited number of Monte Carlo statistics. An uncertainty on $f_{\Lambda_{\mathrm{b}}}$ of $\pm \mathbf{0 . 0 1 9}$ is assigned for the systematic uncertainty and is shown, with all other sources, in table 3.

\section{8 b-flavour tagging}

Systematic uncertainties for b-tagging account for the uncertainty in the evaluation of the efficiency using a finite Monte Carlo sample and for the difference observed between data and Monte Carlo, which is obtained using a double-tag technique [17]. The b-tagging requirement has an efficiency of $0.406 \pm 0.008$ in retaining $\Lambda_{\mathrm{b}} \rightarrow \Lambda \mathrm{X}$ events in the Monte Carlo, where the error is purely statistical. For $\Lambda_{\mathrm{b}} \rightarrow \Lambda \ell \mathrm{X}$ events, one can obtain a clean sample without imposing the b-tagging requirement using the wrong-sign background subtraction [8, 9]. Hence, the efficiency can be measured in both the data and the Monte Carlo simulation for $\Lambda_{\mathrm{b}} \rightarrow \Lambda \ell \mathrm{X}$ events. The efficiency for $\Lambda_{\mathrm{b}} \rightarrow \Lambda \ell \mathrm{X}$ events is measured to be $0.383 \pm 0.032$ for Monte Carlo and $0.426 \pm 0.067$ for data. Applying the b-tagging requirements to a larger sample of Monte Carlo $\Lambda_{\mathrm{b}}$ events reveals that the b-tagging efficiency is slightly less for semileptonic $\Lambda_{\mathrm{b}}$ events than for all $\Lambda_{\mathrm{b}}$ events. The reduced efficiency for semileptonic events is explained by the lower track multiplicity at the $\Lambda_{\mathrm{b}}$ decay vertex because of the invisible neutrino. An additional systematic uncertainty of 0.015 is added in quadrature to account for a small difference in b-tagging efficiency observed between data and Monte Carlo when using double tags. In this case, the b-tagging efficiency for $\Lambda_{\mathrm{b}} \rightarrow \Lambda \mathrm{X}$ events is $0.406 \pm 0.017$ and $0.383 \pm 0.035$ for $\Lambda_{\mathrm{b}} \rightarrow \Lambda \ell \mathrm{X}$ events. For $\mathrm{B} \rightarrow \Lambda \mathrm{X}$ events, we estimate the efficiency from the Monte Carlo to be $0.455 \pm \mathbf{0 . 0 1 9}$, including all systematic uncertainties. The $\mathrm{b}$ tagging efficiency is slightly less for $\Lambda_{\mathrm{b}} \rightarrow \Lambda \mathrm{X}$ events than for other $\mathrm{b} \overline{\mathrm{b}}$ events due to the shorter $\Lambda_{\mathrm{b}}$ lifetime and a lower track multiplicity at the $\Lambda_{\mathrm{b}}$ decay vertex since tracks from the $\Lambda$ decay products do not contribute to the $b$ vertex reconstruction. All b-tagging efficiencies given above are efficiencies per event when one hemisphere contains the specified type of events and the other hemisphere contains any type of b decay.

\section{$6.9 \Lambda$ finding efficiencies}

To evaluate $f\left(\mathrm{~b} \rightarrow \Lambda_{\mathrm{b}}\right) \cdot \mathrm{BR}\left(\Lambda_{\mathrm{b}} \rightarrow \Lambda \mathrm{X}\right)$ and $f(\mathrm{~b} \rightarrow \mathrm{B}) \cdot \mathrm{BR}(\mathrm{B} \rightarrow \Lambda \mathrm{X})$, the systematic uncertainty on the $\Lambda$ finding efficiency is also needed, which is evaluated by comparing data with Monte Carlo. We obtain the $\Lambda$ finding efficiency from the number of $\Lambda$ 's retained in the signal region after applying the $\mathrm{d} E / \mathrm{d} x$ selection criteria for the proton and pion after background subtraction, which is the dominant effect. The background is evaluated using the side-bands of the $\Lambda$ invariant mass distribution. A similar comparison is done after degrading the tracking resolution in the Monte Carlo simulation. We assign an uncertainty of $\pm 2 \%$ to account for both of these effects.

\subsection{Summary of systematic uncertainties}

After making the corrections for systematic shifts listed in table 3 , and including all systematic errors, the composition of the data sample selected with a direct $\Lambda$ and a companion baryon is: 


\begin{tabular}{|c|c|c|c|c|c|c|}
\hline Source & $f_{\Lambda_{\mathrm{b}}}$ shift & $\delta f_{\Lambda_{\mathrm{b}}}$ & $f_{\mathrm{B}}$ shift & $\delta f_{\mathrm{B}}$ & $f_{\text {frag }}$ shift & $\delta f_{\text {frag }}$ \\
\hline$\Lambda$ 's from $\Lambda_{\mathrm{b}}$ decays & $+\mathbf{0 . 0 4 1}$ & $\pm \mathbf{0 . 0 2 1}$ & $+\mathbf{0 . 0 0 6}$ & $\pm \mathbf{0 . 0 0 2}$ & $-\mathbf{0 . 0 4 7}$ & $\pm \mathbf{0 . 0 2 3}$ \\
fragmentation simulation & $-\mathbf{0 . 0 0 5}$ & $\pm \mathbf{0 . 0 0 3}$ & $+\mathbf{0 . 0 2 1}$ & $\pm \mathbf{0 . 0 1 0}$ & $\mathbf{- 0 . 0 1 6}$ & $\pm \mathbf{0 . 0 0 9}$ \\
$\Lambda$ 's from B $\rightarrow \Lambda \mathrm{X}$ & & $\pm \mathbf{0 . 0 0 0}$ & & $\pm \mathbf{0 . 0 1 1}$ & & $\pm \mathbf{0 . 0 1 1}$ \\
protons in B $\rightarrow \Lambda \mathrm{X}$ & & $\pm \mathbf{0 . 0 1 2}$ & & $\pm \mathbf{0 . 0 1 4}$ & & $\pm \mathbf{0 . 0 0 3}$ \\
b hadron momentum & & $\pm \mathbf{0 . 0 0 3}$ & & $\pm \mathbf{0 . 0 0 6}$ & & $\pm \mathbf{0 . 0 0 7}$ \\
fake $\Lambda$ rate in data & & $\pm \mathbf{0 . 0 0 2}$ & & $\pm \mathbf{0 . 0 0 2}$ & & $\pm \mathbf{0 . 0 0 5}$ \\
Monte Carlo sample size & & $\pm \mathbf{0 . 0 1 9}$ & & $\pm \mathbf{0 . 0 1 2}$ & & $\pm \mathbf{0 . 0 1 9}$ \\
\hline total contribution & $+\mathbf{0 . 0 3 6}$ & $\pm \mathbf{0 . 0 3 1}$ & $+\mathbf{0 . 0 2 7}$ & $\pm \mathbf{0 . 0 2 4}$ & $\mathbf{- 0 . 0 6 3}$ & $\pm \mathbf{0 . 0 3 4}$ \\
\hline
\end{tabular}

Table 3: Contributions from different sources to the systematic uncertainties in the estimate of $f_{\Lambda_{\mathrm{b}}}, f_{\mathrm{B}}$ and $f_{\text {frag. }}$. The shifts correspond to the corrections applied to the Monte Carlo when a significant difference is found between data and Monte Carlo.

$$
\begin{gathered}
f_{\Lambda_{\mathrm{b}}}=0.396 \pm 0.046 \pm 0.031 \\
f_{\mathrm{B}}=0.219 \pm 0.032 \pm 0.024, \text { and } \\
f_{\text {frag }}=0.385 \pm 0.047 \pm 0.034
\end{gathered}
$$

Using these fractions, we can adequately reproduce the shape of the momentum distributions observed in data for $\Lambda$ 's and the companion baryons. This can be seen in Figure 5 where we have superimposed the momentum distributions from the three types of Monte Carlo events used to perform the fit.

To verify that no correlations in the two-dimensional distributions have been neglected, we also examine the $\Lambda$ momentum in the data for low and high-momentum companion baryons separately. Again, good agreement between the spectra in data and the total Monte Carlo prediction supports the reliability of this technique.

\section{Calculation of the ratio of branching ratios}

To calculate the ratio of branching ratios, the respective selection efficiencies for $\Lambda \mathrm{e}$ and $\Lambda \mu$ $\left(\epsilon_{\Lambda e}\right.$ and $\left.\epsilon_{\Lambda \mu}\right)$, and the efficiency to find both the $\Lambda$ and the companion baryon $\left(\epsilon_{\Lambda_{\overline{\mathrm{P}}}}\right)$, need to be included. The ratio $R_{\Lambda \ell}$ is given by:

$$
R_{\Lambda \ell}=\frac{\operatorname{BR}\left(\Lambda_{\mathrm{b}} \rightarrow \Lambda \ell \mathrm{X}\right)}{\operatorname{BR}\left(\Lambda_{\mathrm{b}} \rightarrow \Lambda \mathrm{X}\right)}=\frac{1}{2}\left(\frac{N_{\Lambda e}}{\epsilon_{\Lambda e}}+\frac{N_{\Lambda \mu}}{\epsilon_{\Lambda \mu}}\right) \cdot \frac{\epsilon_{\Lambda_{\overline{\mathrm{p}}}}}{f_{\Lambda_{\mathrm{b}}} N} \frac{\epsilon_{\mathrm{b}-\mathrm{tag}}^{\Lambda}}{\epsilon_{\mathrm{b}-\mathrm{tag}}^{\Lambda \ell}} .
$$

$N_{\Lambda e}\left(N_{\Lambda \mu}\right)$ is obtained by subtracting the wrong-sign $\Lambda \mathrm{e}(\Lambda \mu)$ from the right-sign combinations found in data and correcting for the background imbalance. The numerator in $R_{\Lambda \ell}$ is obtained from the average of $N_{\Lambda e}$ and $N_{\Lambda \mu}$, weighted by their respective efficiencies. The number of selected $\Lambda_{\mathrm{b}} \rightarrow \Lambda \mathrm{X}$ events is given by the $\Lambda_{\mathrm{b}}$ fraction $f_{\Lambda_{\mathrm{b}}}$ from the fit times the number of selected events, $N$. The b-tagging efficiencies for events with $\Lambda_{\mathrm{b}} \rightarrow \Lambda \mathrm{X}$ and $\Lambda_{\mathrm{b}} \rightarrow \Lambda \ell \mathrm{X}$ decays (as discussed in section 6.8 ) are given by $\epsilon_{\mathrm{b}-\text { tag }}^{\Lambda}$ and $\epsilon_{\mathrm{b}-\text { tag }}^{\Lambda \ell}$, respectively. Monte Carlo predicts $\epsilon_{\Lambda e}=0.0386 \pm 0.0015$ and $\epsilon_{\Lambda \mu}=0.0423 \pm 0.0016$. This includes the 

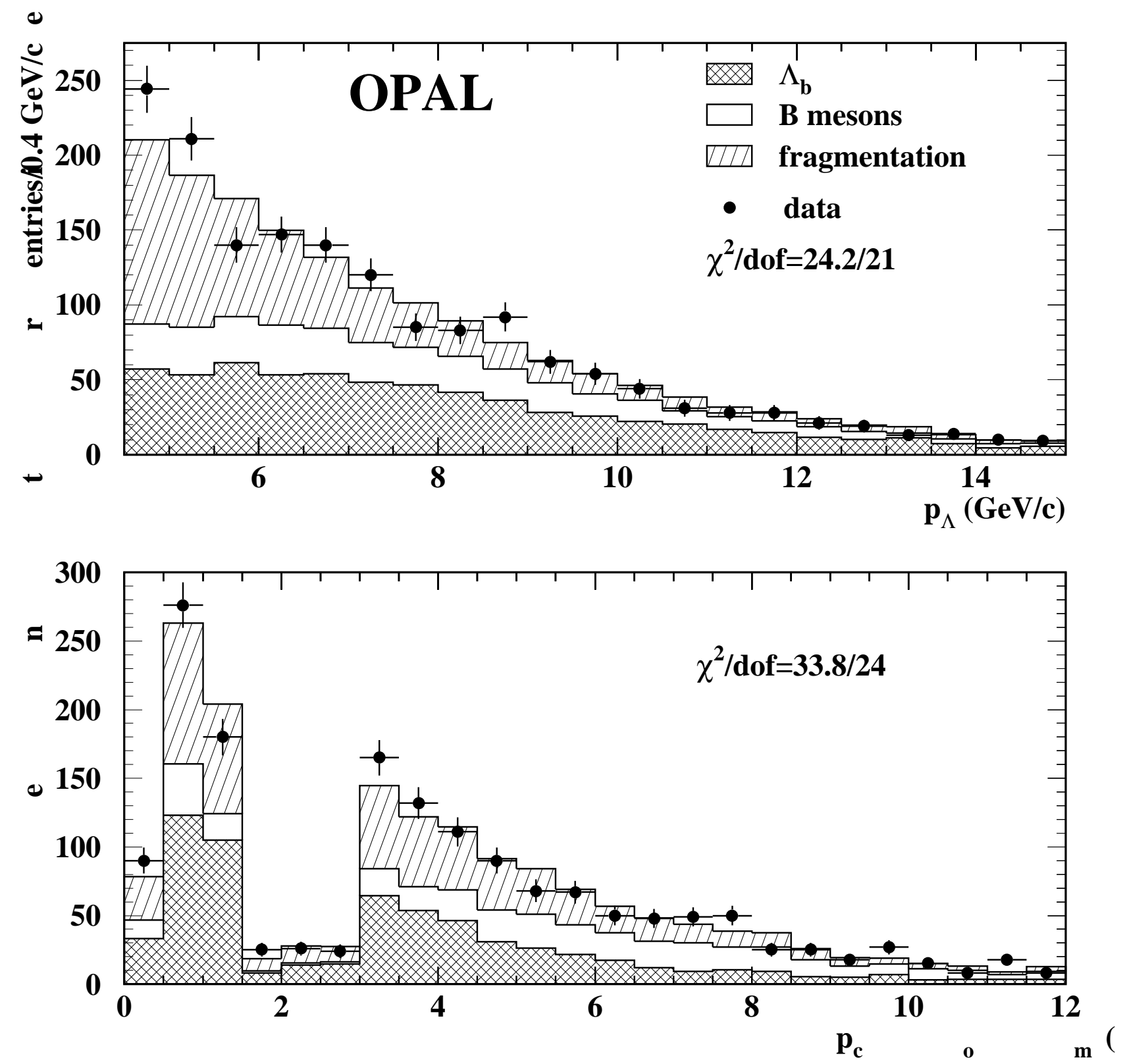

Figure 5: Results of the fit where the contributions from the three types of Monte Carlo events are compared with data for the $\Lambda$ and companion baryon momentum spectra. These values of $\chi^{2}$ are only indicators of the goodness-of-fit and are not used in the optimisation process. 


\begin{tabular}{|c|c|}
\hline$N_{\Lambda e}$ & $64.8 \pm 11.5$ (stat.) \pm 5.0 (syst.) \\
\hline$N_{\Lambda \mu}$ & $80.3 \pm 12.8$ (stat. $) \pm 6.2$ (syst. $)$ \\
\hline$N$ & $1595 \pm 40$ (stat.) \\
\hline$f_{\Lambda_{\mathrm{b}}}$ & $0.396 \pm 0.046($ stat. $) \pm 0.031$ (syst.) \\
\hline$\epsilon_{\Lambda e}$ & $0.0386 \pm 0.0018$ (syst.) \\
\hline$\epsilon_{\Lambda \mu}$ & $0.0423 \pm 0.0020$ (syst.) \\
\hline$\epsilon_{\Lambda_{\overline{\mathrm{P}}}}$ & $0.0233 \pm 0.0007$ (syst.) \\
\hline$\epsilon_{\mathrm{b}-\mathrm{tag}}^{\Lambda}$ & $0.406 \pm 0.017$ (syst.) \\
\hline$\epsilon_{\mathrm{b}-\operatorname{tag}}^{\Lambda \ell}$ & $0.383 \pm 0.035$ (syst.) \\
\hline$R_{\Lambda \ell}$ & $(7.0 \pm 1.2$ (stat. $) \pm 0.7$ (syst. $)) \%$ \\
\hline
\end{tabular}

Table 4: Results for the 1991-95 data sample including all corrections and systematic uncertainties. The numbers of $\Lambda \ell$ have been corrected for the background imbalance described in section 4.1 and the systematic uncertainties come from that correction factor. The correction factor accounting for differences in proton finding efficiencies in the data has been absorbed in $\epsilon_{\Lambda_{\overline{\mathrm{p}}}}$.

$\Lambda$ finding and lepton identification efficiencies. For the denominator, $\epsilon_{\Lambda_{\overline{\mathrm{p}}}}$ is $\mathbf{0 . 0 2 4 2} \pm \mathbf{0 . 0 0 0 4}$ for the $\Lambda$ and companion finding efficiencies combined. All uncertainties are statistical and are estimated from the Monte Carlo.

Only factors not common to the numerator and denominator need to be considered in evaluating the systematic uncertainty. These are uncertainties related to lepton identification for the numerator, and companion baryon finding for the denominator. They reflect differences in observed efficiencies between Monte Carlo and data. For electron identification using a neural network, the systematic uncertainty on the electron finding efficiency is evaluated to be $2.5 \%$ using electrons coming from photo conversions [21]. For muons, the uncertainty is $3.0 \%$ [27]. About $87 \%$ of the companion baryons are protons identified using $\mathrm{d} E / \mathrm{d} x$ information. A difference in efficiency between data and Monte Carlo is observed when the $\mathrm{d} E / \mathrm{d} x$ information is used to identify protons coming from $\Lambda$ decays. The efficiency is $(3.42 \pm 0.05) \%$ higher in the Monte Carlo than in data. A correction of $-3.42 \%$ is applied to $\epsilon_{\Lambda_{\overline{\mathrm{P}}}}$ with a systematic uncertainty equivalent to half the correction itself. The resulting efficiencies and their uncertainties are summarised in table 4.

No systematic uncertainty is attributed to the estimation of the $\Lambda$ finding efficiency. This is because the $\Lambda$ momentum distribution is very similar for $\Lambda_{\mathrm{b}} \rightarrow \Lambda \mathrm{X}$ and $\Lambda_{\mathrm{b}} \rightarrow \Lambda \ell \mathrm{X}$ events, both in data and Monte Carlo. Hence this factor cancels out when taking the ratio.

This leads to a value of

$$
\left.R_{\Lambda \ell}=\frac{\operatorname{BR}\left(\Lambda_{\mathrm{b}} \rightarrow \Lambda \ell \mathrm{X}\right)}{\operatorname{BR}\left(\Lambda_{\mathrm{b}} \rightarrow \Lambda \mathrm{X}\right)}=(7.0 \pm 1.2 \text { (stat.) } \pm 0.7 \text { (syst. })\right) \%
$$

for the ratio of branching ratios averaging over electrons and muons. 


\begin{tabular}{|c|c|}
\hline minimum $\Lambda$ momentum cut & $R_{\Lambda \ell}$ \\
\hline $4.00 \mathrm{GeV} / c$ & $(8.1 \pm 1.4) \%$ \\
$4.25 \mathrm{GeV} / c$ & $(8.0 \pm 1.4) \%$ \\
$4.50 \mathrm{GeV} / c$ & $(7.0 \pm 1.2) \%$ \\
$4.75 \mathrm{GeV} / c$ & $(7.2 \pm 1.3) \%$ \\
$5.00 \mathrm{GeV} / c$ & $(6.3 \pm 1.1) \%$ \\
$5.25 \mathrm{GeV} / c$ & $(6.8 \pm 1.3) \%$ \\
$5.50 \mathrm{GeV} / c$ & $(6.8 \pm 1.3) \%$ \\
\hline
\end{tabular}

Table 5: Effect of the minimum momentum cut on the central value for $R_{\Lambda \ell}$. The errors shown are the statistical uncertainties related to the data sample size and are partly correlated.

\section{Further consistency checks}

The effect of changing the minimum $\Lambda$ momentum requirement on the measured value for $R_{\Lambda \ell}$ is investigated over the range of reliability of the fitting procedure. We increase the minimum $\Lambda$ momentum cut $p_{\text {cut }}$ in steps of $0.25 \mathrm{GeV} / c$ and recalculate $R_{\Lambda \ell}$ each time. All results are consistent with each other, although the values obtained for $p_{\text {cut }}<4.5 \mathrm{GeV} / c$ show a larger deviation. Further investigation reveals that these are consistent with being caused by small statistical fluctuations in the evaluation of the denominator for $R_{\Lambda \ell}$. Similar fluctuations were also observed with Monte Carlo. As $p_{\text {cut }}$ is lowered, the sample composition changes since more background events are introduced, modifying the overall correlations between the fit variables. The different values obtained for $R_{\Lambda \ell}$ seen in table 5 have an r.m.s. value of $0.6 \%$, which is well within the statistical error of this measurement. The 4.5 $\mathrm{GeV} / c$ minimum $\Lambda$ momentum requirement optimises the suppression of contributions from fragmentation and other backgrounds, without causing an increase in the overall statistical uncertainty.

Three uncorrelated and statistically independent subsamples are used to further check our result for the ratio $R_{\Lambda \ell}=\mathrm{BR}\left(\Lambda_{\mathrm{b}} \rightarrow \Lambda \ell \mathrm{X}\right) / \mathrm{BR}\left(\Lambda_{\mathrm{b}} \rightarrow \Lambda \mathrm{X}\right)$ for consistency. In the first test, the data are divided into two subsamples corresponding to the 1991-93 and 1994-95 data samples. In the second test, the sample of all selected $\Lambda_{\mathrm{b}} \rightarrow \Lambda \mathrm{X}$ candidates in the data is divided between events containing a $\Lambda$ versus events containing a $\bar{\Lambda}$ in the denominator while the original full sample is used in the numerator. In the third test, the data are divided in the numerator into the separate electron and muon samples, keeping the original full sample in the denominator. For each of these subsamples, the ratio $R_{\Lambda \ell}$ is calculated. All results are found to be statistically consistent with each other and with the full data sample as can be seen in table 6 .

A separate cross-check is made using this measurement to derive $f\left(\mathrm{~b} \rightarrow \Lambda_{\mathrm{b}}\right) \cdot \mathrm{BR}\left(\Lambda_{\mathrm{b}} \rightarrow\right.$ $\Lambda \ell \bar{\nu} \mathrm{X})$. We measure $(2.57 \pm 0.23) \times 10^{-3}$, to be compared to $(2.91 \pm 0.23 \pm 0.25) \times 10^{-3}$ obtained in a previous analysis [9]. The uncertainty shown is statistical only. The systematic uncertainty would be of similar size. The statistical and systematic uncertainties of the two measurements are only partly correlated since different selection criteria as well as different Monte Carlo simulations and data samples are used for these analyses. 


\begin{tabular}{|c|c|c|c|c|c|c|c|}
\hline & & & & \multicolumn{2}{|c|}{ for denominator: } & \multicolumn{2}{c|}{ for numerator: } \\
& all data & $1991-93$ & $1994-95$ & $\Lambda$ only & $\bar{\Lambda}$ only & e only & $\mu$ only \\
\hline$N_{\Lambda_{\mathrm{b}} \rightarrow \Lambda \ell \mathrm{X}}$ & 145.1 & $\mathbf{6 1 . 6}$ & 83.5 & 145.1 & 145.1 & 64.8 & 80.3 \\
$\Delta N_{\Lambda_{\mathrm{b}} \rightarrow \Lambda \ell \mathrm{X}}$ & \pm 17.3 & \pm 11.0 & \pm 13.3 & \pm 17.3 & \pm 17.3 & \pm 11.5 & \pm 12.8 \\
$N_{\Lambda}$ & 1595 & 698 & 897 & 706 & 889 & 1595 & 1595 \\
$f_{\Lambda_{\mathrm{b}}}$ from fit & $\mathbf{0 . 3 9 6}$ & $\mathbf{0 . 3 5 8}$ & $\mathbf{0 . 4 3 1}$ & $\mathbf{0 . 3 5 0}$ & $\mathbf{0 . 4 3 6}$ & $\mathbf{0 . 3 9 6}$ & $\mathbf{0 . 3 9 6}$ \\
$\Delta f_{\Lambda_{\mathrm{b}}}$ (stat.) & $\pm \mathbf{0 . 0 4 6}$ & $\pm \mathbf{0 . 0 6 8}$ & $\pm \mathbf{0 . 0 6 2}$ & $\pm \mathbf{0 . 0 6 7}$ & $\pm \mathbf{0 . 0 6 4}$ & $\pm \mathbf{0 . 0 4 6}$ & $\pm \mathbf{0 . 0 4 6}$ \\
\hline$R_{\Lambda \ell}$ & $7.0 \%$ & $7.5 \%$ & $\mathbf{6 . 6 \%}$ & $8.1 \%$ & $\mathbf{6 . 2 \%}$ & $\mathbf{6 . 6 \%}$ & $7.4 \%$ \\
stat. error & $\pm 1.2 \%$ & $\pm \mathbf{2 . 0 \%}$ & $\pm 1.4 \%$ & $\pm 1.9 \%$ & $\pm 1.2 \%$ & $\pm 1.4 \%$ & $\pm 1.5 \%$ \\
\hline$\chi^{2}$ /d.o.f. & $24.9 / 33$ & $20.9 / 33$ & $59.4 / 30$ & $47.4 / 33$ & $64.2 / 30$ & $24.9 / 33$ & $24.9 / 33$ \\
\hline
\end{tabular}

Table 6: Results for different data subsamples: Using data from 1991-93 versus data from 1994-95; using exclusively $\Lambda$ or $\bar{\Lambda}$ in the denominator of eq. 7 ; or using electrons and muons separately in the numerator. Here $N_{\Lambda_{\mathrm{b}} \rightarrow \Lambda \ell \mathrm{X}}$ denotes the sum of the electron and muon channels, and $\Delta N_{\Lambda_{\mathrm{b}} \rightarrow \Lambda \ell \mathrm{X}}$, the statistical error. The imbalance in the number of $\Lambda$ and $\bar{\Lambda}$ found reflects the fact that more companion protons than companion anti-protons are found, due to secondary interactions with the detector material. The $\chi^{2} /$ d.o.f. shown are only an indicator of the goodness-of-fit for the fit for the denominator. All results are consistent with each other, as well as with the full data set.

\section{Related measurements and conclusions}

We have presented the first measurement of $R_{\Lambda \ell}=\mathrm{BR}\left(\Lambda_{\mathrm{b}} \rightarrow \Lambda \ell \mathrm{X}\right) / \mathrm{BR}\left(\Lambda_{\mathrm{b}} \rightarrow \Lambda \mathrm{X}\right)$ where $\Lambda_{\mathrm{b}}$ denotes inclusive $\mathrm{b}$ baryons. The measured value is:

$$
R_{\Lambda \ell}=(7.0 \pm 1.2 \pm 0.7) \%
$$

significantly lower than $\mathrm{BR}_{S L}^{B}=(10.43 \pm 0.24) \%[1]$, the semileptonic branching fraction for $\mathrm{B}^{0}$ and $\mathrm{B}^{ \pm}$mesons measured at the $\Upsilon(4 S)$ resonance, as well as significantly lower than $\mathrm{BR}_{S L}^{b}=(11.13 \pm 0.29) \%$ [1] for the average $\mathrm{b}$ hadron semileptonic branching ratio measured at high energy. This indicates that non-spectator amplitudes play a significant role in $b$ hadron decays, as can be inferred from the difference observed between the measured $\mathrm{B}^{0}$ lifetime and the $\mathrm{b}$ baryon lifetime [1].

It is interesting to note that for $\mathrm{B}$ denoting both $\mathrm{B}^{0}$ and $\mathrm{B}^{ \pm}$mesons, and $\mathrm{b}$ denoting all $\mathrm{b}$ hadrons formed at the $\mathrm{Z}^{0}$ resonance

$$
\begin{aligned}
& \frac{R_{\Lambda \ell}}{\mathrm{BR}(\mathrm{B} \rightarrow \ell \mathrm{X})}=0.67 \pm 0.13 \text { and } \frac{\tau_{\Lambda_{b}}}{\tau_{\mathrm{B}}}=0.72 \pm 0.05 \\
& \frac{R_{\Lambda \ell}}{\mathrm{BR}(\mathrm{b} \rightarrow \ell \mathrm{X})}=0.63 \pm 0.13 \text { and } \frac{\tau_{\Lambda_{b}}}{\tau_{\mathrm{b}}}=0.74 \pm 0.05
\end{aligned}
$$

all have very similar values. This indicates that the non-spectator effects responsible for the observed lifetime differences in $\mathrm{b}$ hadrons mainly influence the total decay widths but not the semileptonic decay widths, in accordance with theoretical expectations [5]. 


\begin{tabular}{|c|c|}
\hline$f_{\Lambda_{\mathrm{b}}}$ & $0.396 \pm 0.046 \pm 0.031$ \\
\hline$f_{\mathrm{B}}$ & $0.219 \pm 0.032 \pm 0.024$ \\
\hline$f_{\text {frag }}$ & $0.385 \pm 0.047 \pm 0.034$ \\
\hline$N$ & $1595 \pm 40$ \\
\hline$N_{\text {had }}$ & $3840074 \pm 1960$ \\
\hline$R_{\mathrm{b}}$ & $0.2209 \pm 0.0021$ \\
\hline$\epsilon_{\Lambda_{\bar{p}}}$ & $0.0233 \pm 0.0008($ syst. $)$ \\
\hline$\epsilon_{\mathrm{b}-\mathrm{tag}}^{\Lambda}$ & $0.406 \pm 0.017($ syst. $)$ \\
\hline$\epsilon_{\mathrm{b}-\mathrm{ta}}^{\mathrm{B}}$ & $0.455 \pm 0.019$ (syst. $)$ \\
\hline
\end{tabular}

Table 7: All quantities and their uncertainties needed for the evaluation of the product branching ratios.

From this analysis, we can extract other interesting quantities, provided we include an additional contribution to the systematic uncertainty for the $\Lambda$ selection efficiency, as described in section 6.9. We obtain the product branching fraction:

$$
\begin{gathered}
f\left(\mathrm{~b} \rightarrow \Lambda_{\mathrm{b}}\right) \cdot \mathrm{BR}\left(\Lambda_{\mathrm{b}} \rightarrow \Lambda \mathrm{X}\right)=\frac{f_{\Lambda_{\mathrm{b}}} \cdot N}{\epsilon_{\Lambda_{\overline{\mathrm{p}}}}} \cdot \frac{1}{2 R_{\mathrm{b}} N_{\mathrm{had}}} \text { and } \\
f(\mathrm{~b} \rightarrow \mathrm{B}) \cdot \mathrm{BR}(\mathrm{B} \rightarrow \Lambda \mathrm{X})=\frac{f_{\mathrm{B}} N}{\epsilon_{\Lambda_{\overline{\mathrm{p}}}}} \cdot \frac{1}{2 R_{\mathrm{b}} N_{\text {had }}}
\end{gathered}
$$

where $f\left(\mathrm{~b} \rightarrow \Lambda_{\mathrm{b}}\right)$ is the fraction of $\mathrm{b}$ quarks forming $\Lambda_{\mathrm{b}}$ 's (all weakly decaying b baryons found near the $\mathrm{Z}^{0}$ resonance), and $f(\mathrm{~b} \rightarrow \mathrm{B})$, the fraction of $\mathrm{b}$ quarks forming $\mathrm{B}^{0}, \mathrm{~B}^{ \pm}$and $\mathrm{B}_{\mathrm{s}}$ mesons. $N$ represents the number of selected events containing a direct $\Lambda$ and a companion baryon, $R_{\mathrm{b}}$ is the fraction of hadronic $\mathrm{Z}^{0}$ decays into b $\overline{\mathrm{b}}$ quarks [28], and $N_{\text {had }}$ the number of hadronic $\mathrm{Z}^{0}$ decays passing our selection criteria [15]. The extra factor of two accounts for the presence of two hemispheres per event. All relevant quantities and their systematic uncertainties are summarised in table 7 .

The results are:

$$
\begin{gathered}
f\left(\mathrm{~b} \rightarrow \Lambda_{\mathrm{b}}\right) \cdot \operatorname{BR}\left(\Lambda_{\mathrm{b}} \rightarrow \Lambda \mathrm{X}\right)=(3.93 \pm 0.46 \pm 0.37) \% \text { and } \\
f(\mathrm{~b} \rightarrow \mathrm{B}) \cdot \mathrm{BR}(\mathrm{B} \rightarrow \Lambda \mathrm{X})=(1.94 \pm 0.28 \pm 0.24) \%
\end{gathered}
$$

We obtain the inclusive $\mathrm{b}$ hadron branching ratio using $\left(f_{\Lambda_{\mathrm{b}}}+f_{\mathrm{B}}=1-f_{\text {frag }}\right)$ to evaluate the inclusive fraction of $\mathrm{b}$ hadrons decaying into $\Lambda$ 's.

$$
\mathrm{BR}(\mathrm{b} \rightarrow \Lambda \mathrm{X})=(5.87 \pm 0.46 \pm 0.48) \%
$$

to be compared with $(5.9 \pm 0.7 \pm 0.9) \%$ from DELPHI [29]. The b represents an admixture of $\mathrm{B}^{0}, \mathrm{~B}^{ \pm}, \mathrm{B}_{\mathrm{s}}$ and $\Lambda_{\mathrm{b}}$ as produced near the $\mathrm{Z}^{0}$ resonance. Assuming $f\left(\mathrm{~b} \rightarrow \Lambda_{\mathrm{b}}\right)$ to be $0.132 \pm 0.041$ [1], we obtain 


$$
\operatorname{BR}\left(\Lambda_{\mathrm{b}} \rightarrow \Lambda \mathrm{X}\right)=(29.8 \pm 3.5 \pm 2.3 \pm 9.2) \%
$$

This can be compared with the world average $\left(17_{-6}^{+9} \pm 5\right) \%$ [1]. Both results are evaluated using the same assumption for $f\left(\mathrm{~b} \rightarrow \Lambda_{\mathrm{b}}\right)$. The last uncertainty reflects the uncertainty from $f\left(\mathrm{~b} \rightarrow \Lambda_{\mathrm{b}}\right)$. Assuming $f(\mathrm{~b} \rightarrow \mathrm{B})=0.868 \pm 0.041[1]$ yields

$$
\mathrm{BR}(\mathrm{B} \rightarrow \Lambda \mathrm{X})=(2.2 \pm 0.3 \pm 0.2 \pm 0.1) \%
$$

where the last error comes from the uncertainty on $f(\mathrm{~b} \rightarrow \mathrm{B})$. In this case, $\mathrm{B}$ represents all $\mathrm{B}$ mesons, i.e. an admixture of $\mathrm{B}^{0}, \mathrm{~B}^{ \pm}$and $\mathrm{B}_{\mathrm{s}}$. This result can be compared with the combined results obtained at the $\Upsilon(4 S)$ resonance [1] of $(4.0 \pm 0.5) \%$ which includes only $\mathrm{B}^{0}$ and $\mathrm{B}^{ \pm}$decays.

\section{Acknowledgements:}

We particularly wish to thank the SL Division for the efficient operation of the LEP accelerator and for their continuing close cooperation with our experimental group. In addition to the support staff at our own institutions we are pleased to acknowledge the

Department of Energy, USA, National Science Foundation, USA, Particle Physics and Astronomy Research Council, UK, Natural Sciences and Engineering Research Council, Canada, Israel Science Foundation, administered by the Israel Academy of Science and Humanities, Minerva Gesellschaft,

Japanese Ministry of Education, Science and Culture (the Monbusho) and a grant under the Monbusho International Science Research Program, German Israeli Bi-national Science Foundation (GIF),

Direction des Sciences de la Matière du Commissariat à l'Energie Atomique, France, Bundesministerium für Bildung, Wissenschaft, Forschung und Technologie, Germany, National Research Council of Canada, Hungarian Foundation for Scientific Research, OTKA T-016660, and OTKA F-015089.

\section{References}

[1] Review of Particle Physics, R.M. Barnett et al., Phys. Rev. D 54, 1 (1996).

[2] M. Neubert, C.T. Sachrajda, CERN-TH/96-19 (1996), submitted to Nuclear Physics B.

[3] I.I. Bigi, Il Nuevo Cimento Vol. 109 A, N 6-7 (1996) 713. 
[4] E. Bagan, P. Ball, V.M. Braun, and P. Gosdzinsky, Nucl. Phys. B 432 (1994) 3; Phys. Lett. B 342 (1995) 362. E. Bagan, P. Ball, B. Fiol, and P. Gosdzinsky, Phys. Lett. B 351 (1995) 546.

[5] M. Neubert, Int. J. Mod. Phys. A 11 (1996) 4173.

[6] M.A. Ivanov, V.E. Lyubovitskij, hep-ph/9507374, 1995. Proceedings of the "6th International Conference on Hadron Spectroscopy", Manchester, England, July 1995.

[7] L3 Collab., CERN-PPE/96-49 (1996), submitted to Z. Phys. C.

[8] OPAL Collab., P.D. Acton, et al., Phys. Lett. B 281 (1992) 394;

OPAL Collab., R. Akers, et al., Phys. Lett. B 316 (1993) 435.

[9] OPAL Collab., R. Akers, et al., Z. Phys. C 69 (1996) 195.

[10] OPAL Collab., K. Ahmet et al., Nucl. Inst. and Meth. A 305 (1991) 275;

P.P. Allport et al., Nucl. Inst. and Meth. A 324 (1993) 34;

P.P. Allport et al., Nucl. Inst. and Meth. A 346 (1994) 479.

[11] O. Biebel et al., Nucl. Inst. and Meth. A 323 (1992) 169;

M. Hauschild et al., Nucl. Inst. and Meth. A 314 (1992) 74.

[12] T. Sjöstrand, Comp. Phys. Comm. 82 (1994) 74.

[13] C. Peterson, D. Schlatter, I. Schmitt and. P.M. Zerwas, Phys. Rev. D 27 (1983) 105. The OPAL parameter optimisation for JETSET 7.3 is described in [22].

The OPAL parametrisation for JETSET 7.4 given in

OPAL Collab., G. Alexander, Zeit f. Phys. C 69 (1996) 543.

[14] J. Allison et al., Nucl. Inst. and Meth. A 317 (1992) 47.

[15] OPAL Collab., R.Akers et al., Z. Phys. C 65 (1995) 17.

[16] OPAL Collab., R. Akers et al., Z. Phys. C 63 (1994) 197.

[17] OPAL Collaboration, R. Akers et al., Z. Phys. C 65 (1995) 17.

[18] B. Andersson, G. Gustafson and T. Sjöstrand, Physica Scripta 32 (1985) 574.

[19] OPAL Collab., P. Acton et al., Phys. Lett. B 305 (1993) 415.

[20] OPAL Collab., P.D. Acton et al., Phys. Lett. B 291 (1992) 503.

[21] OPAL Collab., G. Alexander et al., Z. Phys. C70 (1996) 357.

[22] OPAL Collab., P. Acton et al., Z. Phys. C 58 (1993) 523.

[23] OPAL Collab., P. Acton et al., Phys. Lett. B 276 (1992) 379.

[24] T. Mannel and G.A. Schuler, Phys Lett. B 279 (1992) 194.

[25] X.H. Guo and P. Kroll, Z. Phys. C 59 (1993) 567. 
[26] CLEO Collab., G. Crawford et al., Phys. Rev. D 45 (1992) 752; Updated measurement presented in "Inclusive Measurements in B Meson Decay", Bartelt J., to be published in the Proceedings of DPF '96, Minneapolis, MN.

[27] OPAL Collab., K. Ackerstaff et al., CERN-PPE/96-167 submitted to Z. Phys. C.

[28] LEP Experiments : ALEPH, DELPHI, L3, OPAL, Nucl. Inst. and Meth. A 378 (1996) 101.

[29] DELPHI Collab., P. Abreu et al., Phys. Lett. B 347 (1995) 447. 